\title{
Constructive Multiuser Interference in Symbol Level Precoding for the MISO Downlink Channel
}

\author{
Maha Alodeh, Student Member, IEEE, Symeon Chatzinotas, Senior Member, IEEE, \\ Björn Ottersten, Fellow Member, IEEE
}

\begin{abstract}
This paper investigates the problem of interference among the simultaneous multiuser transmissions in the downlink of multiple-antenna systems. Using symbol-level precoding, a new approach to exploit the multiuser interference is discussed. The concept of exploiting the interference between spatial multiuser transmissions by jointly utilizing data information (DI) and channel state information (CSI), in order to design symbol-level precoders, is proposed. To this end, the interference between data streams is transformed under certain conditions into useful signal that can improve the signal to interference noise ratio (SINR) of the downlink transmissions. We propose a maximum ratio transmission (MRT) based algorithm that jointly exploits DI and CSI to glean the benefits from constructive multiuser interference. Subsequently, a relation between the constructive interference downlink transmission and physical layer multicasting is established. In this context, novel constructive interference precoding techniques that tackle the transmit power minimization (min-power) with individual SINR constraints at each user's receivers is proposed. Furthermore, fairness through maximizing the weighted minimum SINR (max-min SINR) of the users is addressed by finding the link between the min power and max min SINR problems. Moreover, heuristic precoding techniques are proposed to tackle the weighted sum rate problem. Finally, extensive numerical results show that the proposed schemes outperform other state of the art techniques.
\end{abstract}

Index Terms-Constructive interference, multiuser MISO, maximum ratio transmission, multicast.

\section{INTRODUCTION}

$\mathbf{I}$ NTERFERENCE is one of the crucial and limiting factors in wireless networks. The idea of utilizing the time and frequency resources has been proposed in the literature to allow different users to share the resources without inducing harmful interference. The concept of exploiting the users' spatial separation has been a fertile research domain for more than one decade. This can be implemented by adding multiple antennas at one or both communication sides. Multiantenna

Maha Alodeh, Symeon Chantzinotas and Björn Ottersten are with Interdisciplinary Centre for Security Reliability and Trust (SnT) at the University of Luxembourg, Luxembourg. E-mails:\{ maha.alodeh@uni.lu, symeon.chatzinotas@uni.lu, and bjorn.ottersten@uni.lu\}.

This work is supported by Fond National de la Recherche Luxembourg (FNR) projects, project Smart Resource Allocation for Satellite Cognitive Radio (SRAT-SCR) ID:4919957 and Spectrum Management and Interference Mitigation in Cognitive Radio Satellite Networks SeMiGod.

Part of this work is accepted for publication in the proceedings of IEEE International Symposium on Information Theory (ISIT), Honolulu-Hawaii, June 2014.

Copyright (c) 2015 IEEE. Personal use of this material is permitted. However, permission to use this material for any other purposes must be obtained from the IEEE by sending a request to pubs-permissions@ieee.org. transceivers empower the communication systems with more degrees of freedom that can boost the performance if the multiuser interference is mitigated properly. Exploiting the space dimension, to serve different users simultaneously in the same time slot and the same frequency band through spatial division multiple access (SDMA), has been investigated in [1]- [8].

The applications of SDMA, in which a single multipleantenna transmitter wants to communicate with multiple receivers, vary according to the requested service. The first service type is known as a broadcast, in which a transmitter has a common message to be sent to multiple receivers. In physical layer research, this service has been studied under the term of physical layer multicasting (i.e. PHY multicasting) [14]- [15]. Since a single data stream is sent to all receivers, there is no multiuser interference. In the remainder of this paper, this case will be referred to as multicast transmission. The second service type is known as unicast, in which a transmitter has an individual message for each receiver. Due to the nature of the wireless medium and the use of multiple antennas, multiple simultaneous unicast transmissions are possible in the downlink of a base station (BS). In these cases, multiple streams are simultaneously sent, which motivates precoding techniques that mitigate the multiuser interference. In information theory terms, this service type has been studied using the broadcast channel [8]. In the remainder of this paper, this case will be referred to as downlink transmission.

In the literature, the precoding techniques for downlink transmission can be further classified as:

1) Group-level precoding in which multiple codewords are transmitted simultaneously but each codeword is addressed to a group of users. This case is also known as multigroup multicast precoding [18]- [21] and the precoder design is dependent on the channels in each user group.

2) User-level precoding in which multiple codewords are transmitted simultaneously but each codeword is addressed to a single user. This case is also known as multiantenna broadcast channel precoding [6]- [13] and the precoder design is dependent on the channels of the individual users. This is a special case of group level precoding where each group consists of a single user.

3) Symbol-level precoding in which multiple symbols are transmitted simultaneously and each symbol is addressed to a single user [22]- [27]. This is also known as a constructive interference precoding and the precoder 
design is dependent on both the channels (CSI) and the symbols of the users (DI).

In the last category, the main idea is to constructively correlate the interference among the spatial streams rather than fully decorrelate them as in the conventional schemes [9]. In [22], the interference in the scenario of BPSK and QPSK is classified into types: constructive and destructive. Based on this classification, a selective channel inversion scheme is proposed to eliminate the destructive interference while leaving the constructive one to be received at the users' terminal. A more advanced scheme is proposed in [23], which rotates the destructive interference to be received as useful signal with the constructive one. These schemes outperform the conventional precodings [9] and show considerable gains. However, the anticipated gains come at the expense of additional complexity at the system design level. Assuming that the channel coherence time is $\tau_{c}$, and the symbol period is $\tau_{s}$, with $\tau_{c} \gg \tau_{s}$ for slow fading channels, the user precoder has to be recalculated with a frequency of $\frac{1}{\tau_{c}}$ in comparison with the symbol based precoder $\frac{1}{\min \left(\tau_{c}, \tau_{s}\right)}=\frac{1}{\tau_{s}}$. Therefore, faster precoder calculation and switching is needed in symbollevel precoding, which can be translated to more expensive hardware. The contributions of this paper can be summarized in the following points:

- A generalized characterization of the constructive interference for any M-PSK is described. Based on this characterization, we propose a new constructive interference precoding scheme, called constructive interference maximum ratio transmissions (CIMRT). This technique exploits the weak points of constructive interference zero forcing precoding (CIZF) in [23].

- We find the relation between the constructive interference precoding problem and PHY layer multicasting and verify it for any M-PSK modulation scenario.

- We propose different symbol-based precoding schemes that aim at optimizing different performance metrics such as minimizing the transmit power while achieving certain SNR targets, maximizing the minimum SNR among the user while keeping the power constraint in the system satisfied and finally maximizing the sum rate of all users without exceeding the permissible amount of power in the system.

The rest of the paper is organized as follows: the channel and system model are explained in Section (II), while Section (III) discusses how conventional downlink precoding techniques tackle interference. Symbol level precoding is described in Section (IV). Moreover, techniques that exploit the multiuser interference in symbol-based precoding are described in Section (V). The relation to PHY-layer multicasting and the solution to the min-power problem are investigated in Section (VI). The problem of maximizing the minimum SINR is tackled in Section (VII). Heuristic sum rate maximization techniques are discussed in Section (VIII). The complexity of the proposed algorithms is discussed in Section (IX). Finally, the performance of the proposed algorithms is evaluated in Section (X).

Notation: We use boldface upper and lower case letters for matrices and column vectors, respectively. $(\cdot)^{H},(\cdot)^{*}$ stand for the Hermitian transpose and conjugate of $(\cdot) . \mathbb{E}(\cdot)$ and $\|\cdot\|$ denote the statistical expectation and the Euclidean norm, $\mathbf{A} \succeq \mathbf{0}$ is used to indicate a positive semidefinite matrix. $\angle(\cdot)$, $|\cdot|$ are the angle and magnitude of $(\cdot)$ respectively. $\mathcal{R}(\cdot), \mathcal{I}(\cdot)$ are the real and the imaginary part of $(\cdot)$. Finally, the vector of all zeros with length of $K$ is defined as $0^{K \times 1}$.

\section{System And Signal Models}

We consider a single-cell multiple-antenna downlink scenario, where a single BS is equipped with $M$ transmit antennas that serves $K$ user terminals, each one of them equipped with a single receiving antenna. The adopted modulation technique is M-PSK. We assume a quasi-static block fading channel $\mathbf{h}_{j} \in \mathbb{C}^{1 \times M}$ between the BS antennas and the $j^{\text {th }}$ user, where the received signal at the $\mathrm{j}^{\text {th }}$ user is written as

$$
y_{j}[n]=\mathbf{h}_{j} \mathbf{x}[n]+z_{j}[n] .
$$

$\mathbf{x}[n] \in \mathbb{C}^{M \times 1}$ is the transmitted signal vector from the multiple-antenna transmitter and $z_{j}$ denotes the noise at the $j^{\text {th }}$ receiver, which is assumed to be an i.d.d. complex Gaussian distributed variable $\mathcal{C N}(0,1)$. A compact formulation of the received signal at all users' receivers can be written as

$$
\mathbf{y}[n]=\mathbf{H} \mathbf{x}[n]+\mathbf{z}[n] .
$$

Let $\mathbf{x}[n]$ be written as $\mathbf{x}[n]=\sum_{j=1}^{K} \mathbf{w}_{j}[n] d_{j}[n]$, where $\mathbf{w}_{j}$ is the $\mathbb{C}^{M \times 1}$ unit power precoding vector for the user $j$. The received signal $y_{j}$ at $j^{\text {th }}$ user in the $n^{t h}$ symbol period is given by

$$
\begin{aligned}
y_{j}[n] & =\sqrt{p_{j}[n]} \mathbf{h}_{j} \mathbf{w}_{j}[n] d_{j}[n] \\
& +\sum_{k \neq j} \sqrt{p_{k}[n]} \mathbf{h}_{j} \mathbf{w}_{k}[n] d_{k}[n]+z_{j}[n]
\end{aligned}
$$

where $p_{j}$ is the allocated power to the $j^{t h}$ user. A more detailed compact system formulation is obtained by stacking the received signals and the noise components for the set of $\mathrm{K}$ selected users as

$$
\mathbf{y}[n]=\mathbf{H W}[n] \mathbf{P}^{\frac{1}{2}}[n] \mathbf{d}[n]+\mathbf{z}[n]
$$

with $\mathbf{H}=\left[\mathbf{h}_{1}^{T}, \ldots, \mathbf{h}_{K}^{T}\right]^{T} \in \mathbb{C}^{K \times M}, \mathbf{W}=\left[\mathbf{w}_{1}, \ldots, \mathbf{w}_{K}\right] \in$ $\mathbb{C}^{M \times k}$ as the compact channel and precoding matrices. Notice that the transmitted signal $\mathbf{d} \in \mathbb{C}^{K \times 1}$ includes the uncorrelated data symbols $d_{k}$ for all users with $\mathbb{E}\left[\left|d_{k}\right|^{2}\right]=1 . \mathbf{P}^{\frac{1}{2}}[n]$ is the power allocation matrix $\mathbf{P}^{\frac{1}{2}}[n]=$ $\operatorname{diag}\left(\sqrt{p_{1}[n]}, \ldots, \sqrt{p_{K}[n]}\right)$. It should be noted that CSI and DI are available at the transmitter side.

\section{Conventional Multiuser Precoding TECHNIQUES}

The main goal of transmit beamforming is to increase the signal power at the intended user and mitigate the interference to non-intended users. This can be obtained by precoding the transmitted symbols in a way that optimizes the spatial directions of the simultaneous transmissions by means of beamforming. This can be mathematically translated to a design problem that targets beamforming vectors to have maximal inner products with the intended channels and minimal 
inner products with the non-intended ones. There are several proposed beamforming techniques in the literature. One of the simplest approaches is to encode the transmitted signal by premultiplying it with the pseudo inverse of the multiuser matrix channel. Several approaches have been proposed including minimizing the sum power while satisfying a set of SINR constraints [6] and maximizing the jointly achievable SINR margin under a power constraint [7]. In any scenario, the generic received signal can be formulated as

$$
\begin{aligned}
\mathbf{y}[n]= & \mathbf{H} \mathbf{x}[n]+\mathbf{z}[n]=\mathbf{H W}[n] \mathbf{P}^{\frac{1}{2}}[n] \mathbf{d}[n]+\mathbf{z}[n] \\
= & {\left[\begin{array}{cccc}
\underbrace{a_{11}}_{\text {desired }} & \underbrace{a_{12}}_{\text {interference }} & \cdots & \underbrace{a_{1 K}}_{\text {interference }} \\
a_{21} & \underbrace{a_{22}}_{\text {desired }} & \cdots & a_{2 K} \\
\vdots & \vdots & \vdots & \vdots \\
\underbrace{a_{K 1}}_{\text {interference }} & \underbrace{a_{K 2}}_{\text {interference }} & \cdots & \underbrace{a_{K K}}_{\text {desired }}
\end{array}\right]\left[\begin{array}{c}
d_{1} \\
\vdots \\
d_{K}
\end{array}\right]+\mathbf{z}(5) }
\end{aligned}
$$

The corresponding SINR of user $j$ can be expressed as

$$
\gamma_{j}=\frac{p_{j}\left\|\mathbf{h}_{j} \mathbf{w}_{j}\right\|^{2}}{\sum_{i=1, i \neq j}^{K} p_{i}\left\|\mathbf{h}_{j} \mathbf{w}_{i}\right\|^{2}+\sigma^{2}}=\frac{\left|a_{j j}\right|^{2}}{\sum_{i=1, i \neq j}^{K}\left|a_{j i}\right|^{2}+\sigma^{2}} .
$$

This paper tries to go beyond this conventional look at the interference by employing symbol-level precoding. This approach can under certain conditions convert the inner product with the non-intended channels into useful power by maximizing them but with the specific directions to which constructively add-up at each user receivers. Taking into account the I/Q plane of the symbol detection, the constructive interference is achieved by using the interfering signal vector to move the received point deeper into the correct detection region. Considering that each user receives a constructive interference from other users' streams, the received signal can be written as

$$
y_{j}[n]=\sum_{i=1}^{K} \underbrace{\sqrt{p_{j}[n]} \mathbf{h}_{j} \mathbf{w}_{i}[n] d_{i}[n]}_{a_{j i}[n] d_{j}[n]}+z_{j}[n] .
$$

This yields the SINR expression for M-PSK symbols as

$$
\gamma_{j}[n]=\frac{\left\|\sum_{i=1}^{K} \sqrt{p_{j}[n]} \mathbf{h}_{j} \mathbf{w}_{i}[n]\right\|^{2}}{\sigma^{2}}=\frac{\left|\sum_{i=1}^{K} a_{j i}\right|^{2}}{\sigma^{2}} .
$$

Different precoding techniques that redesign the terms $a_{j i}, j \neq i$ to constructively correlate them with $a_{j j}$ are proposed in the next sections (V)-(VIII).

\section{A. Power constraints for user-based and symbol-based pre- codings}

In the conventional user-based precoding, the transmitter needs to precode every $\tau_{c}$ which means that the power constraint has to be satisfied along the coherence time $\mathbb{E}_{\tau_{c}}\left\{\|\mathbf{x}\|^{2}\right\} \leq P$. Taking the expectation of $\mathbb{E}_{\tau_{c}}\left\{\|\mathbf{x}\|^{2}\right\}=\mathbb{E}_{\tau_{c}}\left\{\operatorname{tr}\left(\mathbf{W} \mathbf{d} \mathbf{d}^{H} \mathbf{W}^{H}\right)\right\}$, and since $\mathbf{W}$ is fixed along $\tau_{c}$, the previous expression can be reformulated as $\operatorname{tr}\left(\mathbf{W} \mathbb{E}_{\tau_{c}}\left\{\mathbf{d d}^{H}\right\} \mathbf{W}^{H}\right)=\operatorname{tr}\left(\mathbf{W} \mathbf{W}^{H}\right)=\sum_{j=1}^{K}\left\|\mathbf{w}_{j}\right\|^{2}$, where $\mathbb{E}_{\tau_{c}}\left\{\mathbf{d d}^{H}\right\}=\mathbf{I}$ due to uncorrelated symbols over $\tau_{c}$.
However, in symbol-level precoding the power constraint should be guaranteed for each symbol vector transmission, namely for each $\tau_{s}$. In this case the power constraint satisfies $\|\mathbf{x}\|^{2}=\mathbf{W} \mathbf{d d}^{H} \mathbf{W}^{H}=\left\|\sum_{j=1}^{K} \mathbf{w}_{j} d_{j}\right\|^{2}$. In the next sections, we characterize the constructive interference and show how to exploit it in the multiuser downlink transmissions ${ }^{1}$.

\section{Constructive Interference}

Interference is a random deviation which can move the desired constellation point in any direction. To address this problem, the power of interference has been used in the past to regulate its effect on the desired signal point. The interference among the multiuser spatial streams leads to a deviation of the received symbols outside of their detection region. However, in symbol-level precoding (e.g. M-PSK) this interference pushes the received symbols further into the correct detection region and, as a consequence it enhances the system performance. Therefore, interference can be classified into constructive or destructive based on whether it facilitates or deteriorates the correct detection of the received symbol. For BPSK and QPSK scenarios, a detailed classification of interference is discussed thoroughly in [22]. Although BPSK and QPSK are the most used MPSK modulations, the constructive interference pushes the detected constellation point deeper into detection region. Therefore, higher order MPSK modulation can be utilized exploiting constructive interference concept. In this section, we describe the required conditions to have constructive interference for any M-PSK modulation.

\section{A. Constructive Interference Definition}

Assuming both DI and CSI are available at the transmitter, the unit-power created interference from the $k^{t h}$ data stream on the $j^{\text {th }}$ user can be formulated as:

$$
\psi_{j k}=\frac{\mathbf{h}_{j} \mathbf{w}_{k}}{\left\|\mathbf{h}_{j}\right\|\left\|\mathbf{w}_{k}\right\|} .
$$

Since the adopted modulations are M-PSK ones, a definition for constructive interference can be stated as

Lemma 1. An M-PSK modulated symbol $d_{k}$, is said to receive constructive interference from another simultaneously transmitted symbol $d_{j}$ which is associated with $\mathbf{w}_{j}$ if and only if the following inequalities hold

$$
\begin{gathered}
\angle d_{j}-\frac{\pi}{M} \leq \arctan \left(\frac{\mathcal{I}\left\{\psi_{j k} d_{k}\right\}}{\mathcal{R}\left\{\psi_{j k} d_{k}\right\}}\right) \leq \angle d_{j}+\frac{\pi}{M}, \\
\mathcal{R}\left\{d_{k}\right\} \cdot \mathcal{R}\left\{\psi_{j k} d_{j}\right\}>0, \mathcal{I}\left\{d_{k}\right\} \cdot \mathcal{I}\left\{\psi_{j k} d_{j}\right\}>0 .
\end{gathered}
$$

Proof. For any M-PSK modulated symbol, the region of correct detection lies in $\theta_{j} \in\left[\angle d_{j}-\frac{\pi}{M}, \angle d_{j}+\frac{\pi}{M}\right]$, where $\theta_{j}$ is the angle of the detected symbols. In order for the interference to be constructive, the received interfering signal should lie in the region of the target symbol. For the first

\footnotetext{
${ }^{1}$ From now on, we assume that the transmission changes at each symbol and we drop the time index for ease of notation.
} 
condition, the $\arctan (\cdot)$ function checks whether the received interfering signal originating from the $d_{k}{ }^{\text {th }}$ transmit symbol is located in the detection region of the target symbol. However, trigonometric functions are not one-to-one. This means that it manages to check the two quadrants which the interfering symbol may lie in. To find which one of these quadrants is the correct one, an additional constraint is added to check the sign compatibility of the target and received interfering signals.

Corollary 1. The constructive interference between two streams is mutual. If the stream $\mathbf{w}_{j} d_{j}$ constructively interferes with $\mathbf{w}_{k} d_{k}$ (i.e. pushes $d_{k}$ deeper in its detection region), then the interference from transmitting the stream $\mathbf{w}_{k} d_{k}$ is constructive to $d_{j}$.

For constructively interfering symbols, the value of the received signal can be bounded as

$$
\sqrt{p}_{j}\left\|\mathbf{h}_{j}\right\| \stackrel{(a)}{\leq}\left|y_{j}\right| \stackrel{(b)}{\leq}\left\|\mathbf{h}_{j}\right\|\left(\sqrt{p}_{j}+\sum_{\forall k, k \neq j}^{K} \sqrt{p}_{k}\left|\psi_{j k}\right|\right) .
$$

The inequality (a) holds when all simultaneous users are orthogonal (i.e. $\psi_{j k}=0$ ), while (b) holds when all created interference is aligned with the transmitted symbol as $\angle d_{k}=\angle \psi_{j k} d_{j}$ and $\psi_{j k}=0, \angle d_{k}=\angle \psi_{j k} d_{j}$. Eq. (10) indicates that in the case of constructive interference, having fully correlated signals is beneficial as they contribute to received signal power.

In conventional precoding techniques, the previous inequality can be reformulated as

$$
0 \stackrel{(a)}{\leq}\left|y_{j}\right| \stackrel{(b)}{\leq} \sqrt{p_{j}}\left\|\mathbf{h}_{j}\right\|
$$

The worst case scenario can occur when all users are colinear, that is when $\psi_{j k} \rightarrow 1$. The channel cannot be inverted and thus the interference cannot be mitigated. The optimal scenario takes place when all users have physically orthogonal channels which entails no multiuser interference. Therefore, utilizing CSI and DI leads to higher performance in comparison to employing conventional techniques.

\section{Constructive Interference Precoding for MISO DOWNLINK CHANNELS}

In the remainder of this paper, it is assumed that the transmitter is capable of designing symbol-level precoding utilizing both CSI and $\mathrm{DI}^{2}$.

\section{A. Correlation Rotation Zero Forcing Precoding (CIZF)}

The precoder aims at minimizing the mean square error while it takes into account the rotated constructive interference [23]. The optimization problem can be formulated as

$$
\mathcal{J}=\min _{\mathbf{W}} \quad \mathbb{E}\left\{\left\|\mathbf{R}_{\phi} \mathbf{d}-(\mathbf{H W d}+\mathbf{z})\right\|^{2}\right\},
$$

\footnotetext{
${ }^{2}$ From this section, we combine the the precoding design with power allocation
}

where $\mathbf{P}^{\frac{1}{2}}=\mathbf{I}$ in this scenario. The solution can be easily expressed as

$$
\mathbf{W}_{C I Z F}=\gamma \mathbf{H}^{H}\left(\mathbf{H H}^{H}\right)^{-1} \mathbf{R}_{\phi},
$$

where $\gamma=\sqrt{\frac{P}{\operatorname{tr}\left(\mathbf{R}_{\phi}^{H}\left(\mathbf{H H}^{H}\right)^{-1} \mathbf{R}_{\phi}\right)}}$ ensures the power normalization. The cross correlation factor between the $j^{\text {th }}$ user's channel and $k^{t h}$ transmitted data stream can be expressed as

$$
\rho_{j k}=\frac{\mathbf{h}_{j} \mathbf{h}_{k}^{H}}{\left\|\mathbf{h}_{k}\right\|\left\|\mathbf{h}_{j}\right\|} .
$$

The relative phase $\phi_{j k}$ that grants the constructive simultaneous transmissions can be expressed as

$$
\phi_{j k}=\angle d_{j}-\angle\left(\rho_{j k} d_{k}\right) .
$$

The corresponding rotation matrix can be implemented as:

$$
\mathbf{R}_{\phi}(j, k)=\rho_{j k} \exp \left(\phi_{j k} i\right),
$$

and the received signal at the $j^{\text {th }}$ user can be expressed as

$$
y_{j} \stackrel{a}{=} \gamma\left\|\mathbf{h}_{j}\right\|\left(\sum_{k=1}^{K} \rho_{j k} d_{k}\right)+z_{j} \stackrel{b}{=} \gamma\left\|\mathbf{h}_{j}\right\|\left(\sum_{k=1}^{K} \varepsilon_{j k}\right) d+z_{j},
$$

where $\varepsilon_{j k}$ has the same magnitude as $\rho_{j k}$ but with a different phase, and $d: d \in \mathbb{C}^{1 \times 1},|d|=1, \angle d=\theta, \theta \in[0,2 \pi]$. By taking a look at (16-b), it has a multicast formulation since it seems for each user that BS sends the same symbol for all users by applying a user-dependent rotation.

Remark 1. It can be noted that this solution includes a zero forcing step and a correlation step $\mathbf{R}_{\phi}$. The correlation step aims at making the transmitted signals be constructively received at each user. Unfortunately, this design fails when we deal with co-linear users, that is when $\rho_{j k} \rightarrow 1$. However, intuitively having co-linear users should create more constructive interference and higher gain should be anticipated. It can be easily concluded that the source of this contradiction is the zero forcing step. In an effort to overcome the problem, we propose a new precoding technique in the next section.

\section{B. Proposed Constructive Interference Maximum Ratio Trans- mission (CIMRT)}

Maximum ratio transmission (MRT) is not suitable for multiuser downlink transmissions in a MISO system due to the intolerable amount of the created interference. On the other hand, this feature makes it a good candidate for constructive interference. The naive maximum ratio transmission (nMRT) can be formulated as

$$
\mathbf{W}_{\mathrm{nMRT}}=\left[\frac{\mathbf{h}_{1}{ }^{H}}{\left\|\mathbf{h}_{1}\right\|}, \frac{\mathbf{h}_{2}{ }^{H}}{\left\|\mathbf{h}_{2}\right\|}, \ldots, \frac{\mathbf{h}_{K}{ }^{H}}{\left\|\mathbf{h}_{K}\right\|}\right] .
$$

A new look at the received signal can be viewed by exploiting the singular value decomposition of $\mathbf{H}=\mathbf{S V D}$, and $\mathbf{W}_{\text {nMRT }}=\mathbf{D}^{H} \mathbf{V}^{\prime} \mathbf{S}^{H}$ as follows

$$
\begin{aligned}
\mathbf{y} & =\mathbf{H W d}+\mathbf{z}=\mathbf{S V D D}^{H} \mathbf{V}^{\prime} \mathbf{S}^{H} \mathbf{d}+\mathbf{z} \\
& =\mathbf{G B d}+\mathbf{z},
\end{aligned}
$$

where

$$
\mathbf{G}=\mathbf{S V V ^ { \prime }}, \quad \mathbf{B}=\mathbf{S}^{H} .
$$


$\mathbf{S} \in \mathbb{C}^{K \times M}$ is a unitary matrix that contains the left-singular vectors of $\mathbf{H}$, the matrix $\mathbf{V}$ is an $K \times M$ diagonal matrix with nonnegative real numbers on the diagonal, and $\mathbf{D} \in \mathbb{C}^{M \times M}$ contains right-singular vectors of $\mathbf{H}$. $\mathbf{V}^{\prime}$ is the power scaled of $\mathbf{V}$ to normalize each column in $\mathbf{W}_{\text {nMRT }}$ to unit. The received signal can be expressed as

$$
\begin{aligned}
y_{j} & =\left\|\mathbf{h}_{j}\right\|\left(\sum_{k=1}^{K} \rho_{j k} d_{k}\right)+z_{j} \\
& =\left\|\mathbf{g}_{j}\right\| \sum_{k=1}^{K} \xi_{j k} \exp \left(\theta_{k}\right) d+z_{j},
\end{aligned}
$$

where $\mathbf{g}_{j}$ is the $j^{\text {th }}$ row of the matrix $\mathbf{G}, \xi_{j k}=\frac{\mathbf{g}_{j} \mathbf{b}_{k}}{\left\|\mathbf{g}_{j}\right\|}$. Since $\mathbf{B}$ is a unitary matrix, it can have uncoupled rotations which can grant the constructivity of interference.

Let $\mathbf{R}_{k j}$ be the rotation matrix in the $\left(\mathbf{b}_{k}, \mathbf{b}_{j}\right)$-plane, which performs an orthogonal rotation of the $k^{t h}$ and $j^{\text {th }}$ columns of a unitary matrix while keeping the others fixed, thus preserving unitarity. Assume without loss of generality that $k>j$. Givens rotation matrix in the $\left(\mathbf{b}_{k}, \mathbf{b}_{j}\right)$-plane can be defined as

$$
\mathbf{R}_{k j}(\alpha, \delta)=\left[\begin{array}{cccccc}
1 & 0 & \ldots & 0 & \ldots & 0 \\
\vdots & \ddots & \vdots & \vdots & \vdots & \vdots \\
0 & \ldots & \cos \alpha & \ldots & -\sin \alpha e^{-\delta i} & \ldots \\
\vdots & \vdots & \vdots & \vdots & \vdots & \\
\vdots & \vdots & \sin \alpha e^{-\delta i} & \ldots & \cos \alpha & \ldots \\
\vdots & \ldots \ldots & \vdots & \ldots & & 1
\end{array}\right]
$$

where the non-trivial entries appear at the intersections of $k^{\text {th }}$ and $j^{\text {th }}$ rows and columns. Hence, any unitary matrix $\mathbf{B}^{\prime}$ can be expressed using the following parametrization

$$
\mathbf{B}^{\prime}=\mathbf{B} \prod_{j=1}^{K} \prod_{k=j+1}^{K} \mathbf{R}_{k j} .
$$

It can be seen from the structure of the matrix in (20) that rotation in the $\left(\mathbf{b}_{k}, \mathbf{b}_{j}\right)$-plane does not change the directions of the remaining beamforming vectors. Therefore, it just modifies the value of $\xi_{k k}$, and the precoder reads as

$$
\mathbf{W}_{\text {CIMRT }}=\mathbf{D}^{H} \mathbf{V}^{\prime} \mathbf{B}^{\prime} .
$$

To grant constructive interference, we need to rotate the $\left(\mathbf{b}_{k}, \mathbf{b}_{j}\right)$-plane by formulating the rotation as a set of nonlinear equations as

$$
\begin{aligned}
\xi_{k k}^{\prime} d_{k} & =\xi_{k k} \cos (\alpha) d_{k}-\xi_{k j} \sin (\alpha) e^{-i \delta} d_{j} \\
\xi_{j j}^{\prime} d_{j} & =\xi_{j k} \sin (\alpha) e^{i \delta} d_{k}+\xi_{j j} \cos (\alpha) d_{j} .
\end{aligned}
$$

Remark 2. The rotation of the $\left(\mathbf{b}_{k}, \mathbf{b}_{j}\right)$-plane is independent and decoupled from any other plane. This means that any implemented rotation on this plane only affects the $(k, j)$ pair.

Since the set of non-linear equations can have different roots, the function needs to be evaluated at the obtained root in order to find the optimal ones. The optimal solution can be found when solving for $\xi_{k k}^{\prime}=\sqrt{\xi_{k k}^{2}+\xi_{k j}^{2}}, \xi_{j j}^{\prime}=$ $\sqrt{\xi_{j j}^{2}+\xi_{j k}^{2}}$. Sometimes it is not feasible to solve for $\xi_{k k}^{*}$ and $\xi_{j j}^{*}$, and their values need to be reduced correspondingly. The proposed algorithm can be illustrated in the following table

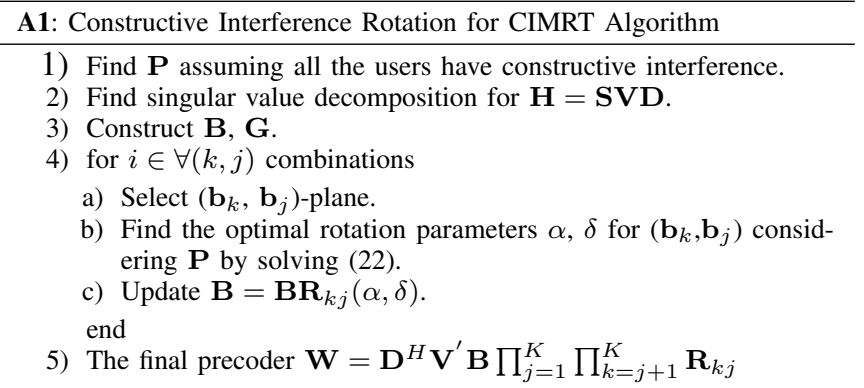

\section{CONSTRUCTIVE INTERFERENCE FOR POWER MINIMIZATION}

\section{A. Constructive Interference Power Minimization Precoding (CIPM)}

According to the definition of constructive interference, we should design the constructive interference precoders by assuring that the sum of the precoders and data symbols in the expression forces the received signal to the detection region of the desired symbol for each user. Therefore, the optimization that minimizes the transmit power and grants the constructive reception of the transmitted data symbols can be written as

$$
\begin{aligned}
\mathbf{w}_{k}\left(d_{j}, \mathbf{H}, \boldsymbol{\zeta}\right) & =\arg \min _{\mathbf{w}_{1}, \ldots, \mathbf{w}_{K}}\left\|\sum_{k=1}^{K} \mathbf{w}_{k} d_{k}\right\|^{2} \\
\text { s.t. } & \left\{\begin{array}{l}
\mathcal{C} 1: \angle\left(\mathbf{h}_{j} \sum_{k=1}^{K} \mathbf{w}_{k} d_{k}\right)=\angle\left(d_{j}\right), \forall j \in K \\
\mathcal{C} 2:\left\|\mathbf{h}_{j} \sum_{k=1}^{K} \mathbf{w}_{k} d_{k}\right\|^{2} \geq \sigma^{2} \zeta_{j} \quad, \forall j \in K,
\end{array}\right.
\end{aligned}
$$

where $\zeta_{j}$ is the SNR target for the $j^{\text {th }}$ user that should be granted by the transmitter, and $\zeta=\left[\zeta_{1}, \ldots, \zeta_{K}\right]$ is the vector that contains all the SNR targets. The set of constraints $\mathcal{C}_{1}$ guarantees that each user receives its corresponding data symbol $d_{j}$. A reformulation for the previous problem (23) using $\hat{\mathbf{w}}_{k}=\mathbf{w}_{k} d_{k}$ can be expressed as

$$
\begin{aligned}
\hat{\mathbf{w}}_{k}\left(d_{j}, \mathbf{H}, \boldsymbol{\zeta}\right)=\arg \min _{\hat{\mathbf{w}}_{1}, \ldots, \hat{\mathbf{w}}_{K}}\left\|\sum_{k=1}^{K} \hat{\mathbf{w}}_{k}\right\|^{2} \\
\text { s.t. } \quad\left\{\begin{array}{l}
\mathcal{C} 1: \angle\left(\mathbf{h}_{j} \sum_{k=1}^{K} \hat{\mathbf{w}}_{k}\right)=\angle\left(d_{j}\right), \forall j \in K \\
\mathcal{C} 2:\left\|\mathbf{h}_{j} \sum_{k=1}^{K} \hat{\mathbf{w}}_{k}\right\|^{2} \geq \sigma^{2} \zeta_{j} \quad, \forall j \in K .
\end{array}\right.
\end{aligned}
$$

The replaced variables $\hat{\mathbf{w}}_{k}$ 's indicate that it is not necessary to send the exact symbols $d_{1}, \ldots, d_{K}$; they can be included in the precoding design as long as they are received correctly at the users' terminals. Then, we design the final output vector $\mathbf{x}$ instead of designing the whole $\mathbf{W}$ with the assumption that $\mathbf{d}$ is fixed. This means that the proposed methods move away from the classical approach of linear beamforming, where the precoding matrix is multiplied with the symbol vector. Instead, we adopt an approach where the transmit signal vector is designed directly based on an optimization problem. 


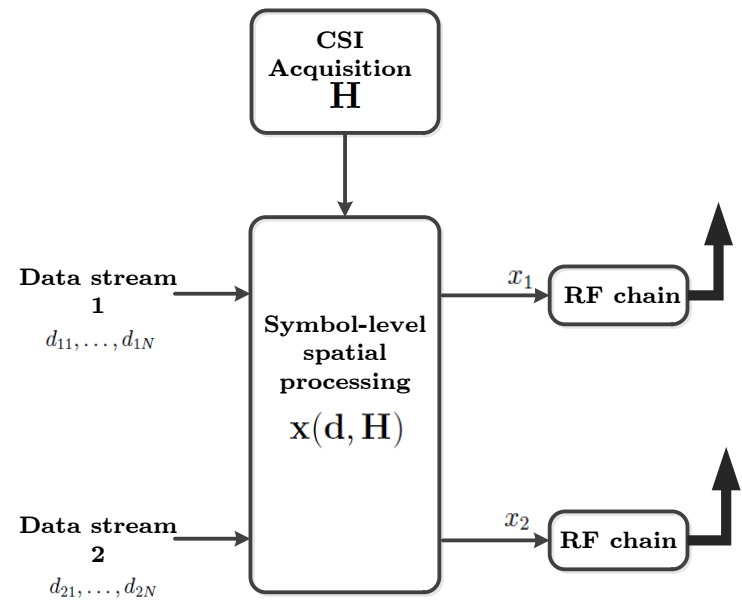

Fig. 2. Symbol-level precoding model in the constructive interference MISO systems. The transmit vector $\mathbf{x}$ is calculated once per symbol.

Lemma 2. Assuming a conventional linear precoder $\mathbf{x}=$ $\mathrm{Wd}$, the transmitted signal vector $\mathrm{x}$ which minimizes the transmit power can be calculated using a unit-rank precoding matrix $\mathbf{W}$.

Proof. This can be proved by using the auxiliary variable $\mathrm{x}=$ $\sum_{k=1}^{K} \hat{\mathbf{w}}_{k}$ and substituting it in the optimization problem (24). The optimization can be rewritten as

$$
\begin{aligned}
\mathbf{x}\left(d_{j}, \mathbf{H}, \boldsymbol{\zeta}\right)= & \arg \min _{\mathbf{x}}\|\mathbf{x}\|^{2} \\
\text { s.t. } & \left\{\begin{array}{l}
\mathcal{C} 1: \angle\left(\mathbf{h}_{j} \mathbf{x}\right)=\angle\left(d_{j}\right), \forall j \in K \\
\mathcal{C} 2:\left\|\mathbf{h}_{j} \mathbf{x}\right\|^{2} \geq \sigma^{2} \zeta_{j}
\end{array}, \forall j \in K .\right.
\end{aligned}
$$

$\mathbf{x}$ is a vector, which makes the solution $\mathbf{W}=\left[\mathbf{w}_{1}, \ldots, \mathbf{w}_{K}\right]$ a unit rank as $\mathbf{w}_{k}=\frac{\mathbf{x}}{K}$, and the virtual input vector satisfies $\mathbf{d}_{v}=\mathbf{1}^{K \times 1}$.

Based on Lemma 2, the differentiation between the conventional and constructive interference precoding techniques is illustrated in Fig. (1)-(2). Fig. (1) shows how the conventional precoding depends only on the CSI information to optimize $\mathbf{W}$ that carry the data symbols $\mathbf{d}$ and without any design dependency between them. Therefore, the transmitted output vector can be formulated as $\mathbf{x}=\sum_{j=1}^{K} \mathbf{w}_{j} d_{j}$. The final output vector $\mathbf{x}$ only depends on the DI and CSI and this dependence is a linear one. On the other hand, in constructive interference precoding schemes, the precoding directly depends on both the CSI and DI information to exploit the interference through skipping the intermediate step (i.e. optimizing $\mathbf{W}$ ) and optimizing directly the vector $\mathbf{x}$. In constructive interference schemes (25), the relation between the data symbols in $\mathbf{d}$ and the final output vector $\mathbf{x}$ cannot be explicitly described as in linear conventional precoding scheme. This can be explained by the fact that the DI is used to design the output vector but is not necessarily physically transmitted as in conventional linear precoding. An implicit set of virtual data is used instead which is explained later in this paper.
B. The Relation Between Constructive Interference Precoding and Constrained Constellation Multicast

By taking a look at Lemma 2, the solution of the optimization problem resembles the solution of multicast problem in which the transmitter sends a single message to users [14]- [17]. However in our problem, we have an additional constraint $\mathcal{C}_{1}$ in (23)-(25) which guarantees that each user detects correctly its symbol based on the received signal.

Theorem 1. The optimal precoder for CIPM

$$
\begin{array}{cl}
\mathbf{x}_{C I P M}(\mathbf{d}, \mathbf{H}, \boldsymbol{\zeta})=\underset{\mathbf{w}}{\arg \min _{\mathbf{w}}} & \operatorname{tr}\left(\mathbf{x} \mathbf{x}^{H}\right) \\
\text { s.t. } & \angle\left(\mathbf{h}_{j} \mathbf{x}\right)=\angle\left(d_{j}\right) \quad \forall j \in K \\
& \mathbf{h}_{j} \mathbf{x} \mathbf{x}^{H} \mathbf{h}_{j}^{H}=\zeta_{j} \quad \forall j \in K(26)
\end{array}
$$

is given by $\mathbf{x}_{e}\left(d, \mathbf{A}\left(d, d_{j}\right) \mathbf{H}\right)$ in (26), where $\mathbf{A}\left(d, d_{j}\right)$

$$
\mathbf{A}\left(d, d_{j}\right)=\left\{\begin{array}{l}
\exp \left(\left(\angle d-\angle d_{j}\right) i\right), \quad j=k \\
0, \quad j \neq k .
\end{array}\right.
$$

Proof. We assume that we have the following equivalent channel as

$$
\mathbf{H}_{e}=\mathbf{A} \mathbf{H} .
$$

The power minimization can be rewritten by replacing $\mathbf{H}$ by its equivalent channel $\mathbf{H}_{e}$ in (26) as

$$
\begin{array}{lll}
\min _{\mathbf{x}_{e}} & \left(\mathbf{x}_{e}^{H} \mathbf{x}_{e}\right) \\
\text { s.t. } & \angle\left(\mathbf{h}_{e, j} \mathbf{x}_{e}\right)=\angle(d) \quad \forall j \in K \\
& \mathbf{h}_{e, j} \mathbf{x}_{e} \mathbf{x}_{e}^{H} \mathbf{h}_{e, j}^{H}=\zeta_{j} \quad \forall j \in K .
\end{array}
$$

where $\mathbf{h}_{e, j}$ is the $j^{t h}$ row of the $\mathbf{H}_{e}$. Rewriting the first constraints in (29) as

$$
\begin{aligned}
& \angle\left(d-d_{j}\right) \angle\left(\mathbf{h}_{j} \mathbf{x}_{e}\right)=\angle(d) \\
\equiv \quad & \angle\left(\mathbf{h}_{j} \mathbf{x}_{e}\right)=\angle\left(d_{j}\right) \quad \forall j \in K
\end{aligned}
$$

shows the equivalence between the constrained constellation multicast channel and constructive interference downlink channel.

By taking a look at (25), the objective function $\|\mathbf{x}\|^{2}$ is unit rank and thereby it is a convex. However, the constraints $\mathcal{C}_{1}$ and $\mathcal{C}_{2}$ are not convex. Therefore, reformulations for $\mathcal{C}_{1}$ and $\mathcal{C}_{2}$ are required.

We can reformulate the problem as

$$
\begin{array}{ll} 
& \mathbf{x}\left(d_{j}, \mathbf{H}, \boldsymbol{\zeta}\right)=\arg \min _{\mathbf{x}}\|\mathbf{x}\|^{2} \\
\text { s.t. } & \left\{\begin{array}{l}
\mathcal{C} 1: \frac{\mathbf{h}_{j} \mathbf{x}-\left(\mathbf{h}_{j} \mathbf{x}\right)^{H}}{i\left(\mathbf{h}_{j} \mathbf{x}+\left(\mathbf{h}_{j} \mathbf{x}\right)^{H}\right)}=\tan \left(d_{j}\right), \forall j \in K \\
\mathcal{C} 2: \mathcal{R}\left\{d_{j}\right\} \cdot \mathcal{R}\left\{\mathbf{h}_{j} \mathbf{x}\right\} \geq 0, \forall j \in K \\
\mathcal{C} 3: \mathcal{I}\left\{d_{j}\right\} \cdot \mathcal{I}\left\{\mathbf{h}_{j} \mathbf{x}\right\} \geq 0, \forall j \in K \\
\mathcal{C} 4:\left\|\mathbf{h}_{j} \mathbf{x}\right\|^{2} \geq \sigma^{2} \zeta_{j}, \forall j \in K .
\end{array}\right.
\end{array}
$$

$\mathcal{C}_{1}$ is the constraint that assures the angle of the received signal is either $\angle d_{j}$ or $\pi+\angle d_{j} . \mathcal{C}_{2}$ and $\mathcal{C}_{3}$ are the constraints that the received signal lies in the phase sector. $\mathcal{C}_{4}$ is to assure that the received signal power is higher than certain target SNR. The minimum transmit power in (23)-(25) occurs when the inequality constraints $\mathcal{C}_{4}$ are replaced by equality. Since there is no constraint on the maximum power, this problem is always feasible. Therefore, the minimum power 


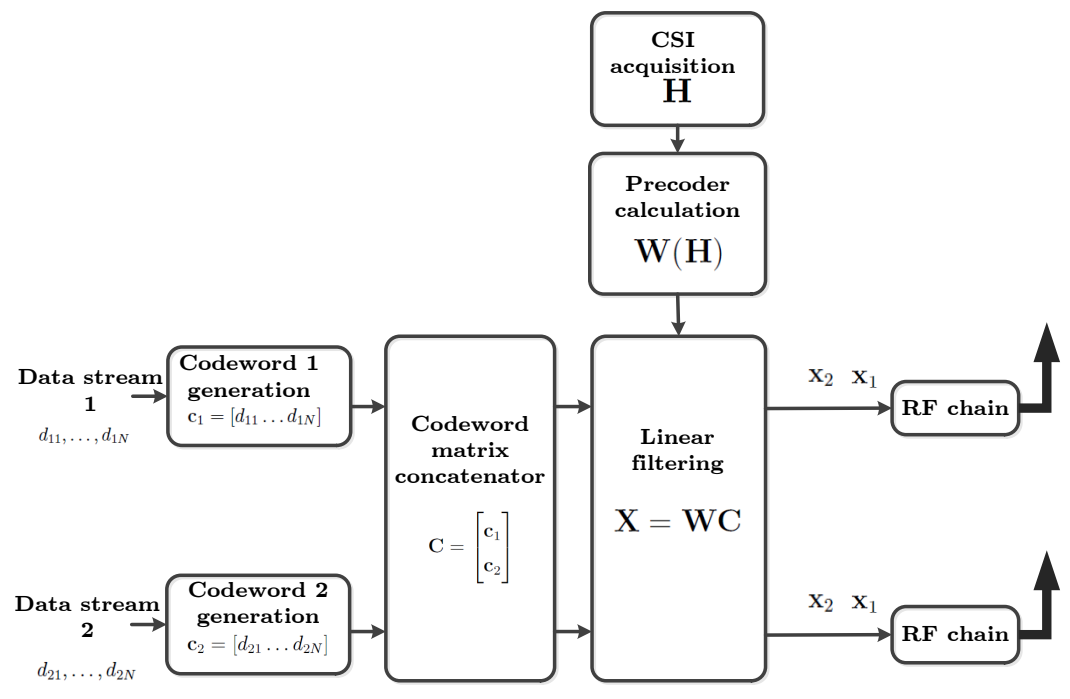

Fig. 1. Codeword-level precoding model in the conventional MISO systems. The precoder is calculated and applied once for the whole codeword since it is independent of the actual symbols.

can be achieved when all users achieve their target SNR exactly. $\mathcal{C}_{1}$ and $\mathcal{C}_{2}$ in (25) can be transformed into affine constraints, which makes the optimization convex and thus solvable through standard methods. A final formulation can be expressed as

$$
\begin{aligned}
& \arg \min _{\mathbf{x}}\|\mathbf{x}\|^{2} \\
& \text { s.t. }\left\{\begin{array}{l}
\mathcal{C} 1: \frac{\mathbf{h}_{j} \mathbf{x}-\left(\mathbf{h}_{j} \mathbf{x}\right)^{H}}{2 i}=\sigma \sqrt{\zeta_{j}} \mathcal{I}\{d\}, \forall j \in K \\
\mathcal{C} 2: \frac{\mathbf{h}_{j} \mathbf{x}+\left(\mathbf{h}_{j} \mathbf{x}\right)^{H}}{2}=\sigma \sqrt{\zeta_{j}} \mathcal{R}\left\{d_{j}\right\}, \forall j \in K .
\end{array}\right.
\end{aligned}
$$

It can be viewed that the constraints in (31) are turned into equalities when signal alignment requirements are met. The Lagrangian function can be derived as follows

$$
\begin{aligned}
& \mathcal{L} \quad(\mathbf{x})=\|\mathbf{x}\|^{2} \\
& +\sum_{j} \mu_{j}\left(-0.5 i\left(\mathbf{h}_{j} \mathbf{x}-\mathbf{x}^{H} \mathbf{h}_{j}^{H}\right)-\sigma \sqrt{\zeta_{j}} \mathcal{I}\left\{d_{j}\right\}\right) \\
& +\sum_{j} \alpha_{j}\left(0.5\left(\mathbf{h}_{j} \mathbf{x}+\mathbf{x}^{H} \mathbf{h}_{j}^{H}\right)-\sigma \sqrt{\zeta_{j}} \mathcal{R}\left\{d_{j}\right\}\right)
\end{aligned}
$$

where $\mu_{j}$ and $\alpha_{j}$ are the Lagrangian dual variables. The derivative for the Lagrangian function can be written as

$$
\frac{d \mathcal{L}(\mathbf{x})}{d \mathbf{x}^{*}}=\mathbf{x}+0.5 i \sum_{j} \mu_{j} \mathbf{h}_{j}^{H}+0.5 \sum_{j} \alpha_{j} \mathbf{h}_{j}^{H} .
$$

By equating this term to zero, $\mathbf{x}_{i}$ can be written as

$$
\begin{aligned}
\mathbf{x} & =-0.5 i \sum_{j=1}^{K} \mu_{j} \mathbf{h}_{j}^{H}-0.5 \sum_{j} \alpha_{j} \mathbf{h}_{j}^{H} \\
& \equiv \sum_{j=1}^{K} \nu_{j} \mathbf{h}_{j}^{H}, \forall i \in K,
\end{aligned}
$$

where $\nu_{j} \in \mathbb{C}=-0.5 i \mu_{j}-0.5 \alpha_{j}$. The optimal values of the Lagrangian variables $\mu_{j}$ and $\alpha_{j}$ can be found by substituting $\mathbf{w}$ in the constraints (32) which results in solving the set of $2 K$ equations (36). The final constrained constellation multicast precoder can be found by substituting all $\mu_{j}$ and $\alpha_{j}$ in (35).
Corollary 2. The CI precoding for power minimization $\mathbf{x}_{C I P M}$ as well as the constrained constellation multicast precoding must span the subspaces of each user's channel.

It can be noted from the formulation of $\mathbf{x}_{C I P M}$ that BS should use the same precoder for all users. This result resembles the multicast approach in which the BS wants to deliver the same message to all users [14]- [15]. However in multicast systems, a different symbol should be detected correctly at each user.

Using (35), we can rewrite the received signal at the $j^{\text {th }}$ receiver as

$$
\begin{aligned}
& y_{j}=\mathbf{h}_{j} \mathbf{x}+z_{j}=\mathbf{h}_{j} \sum_{k=1}^{K} \nu_{k} \mathbf{h}_{k}^{H}+z_{j}
\end{aligned}
$$

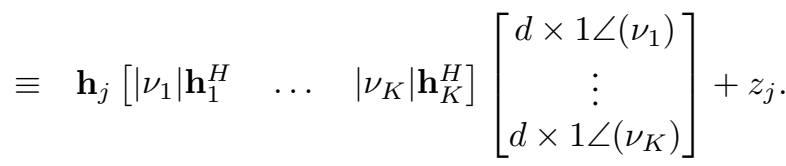

From (37), the constellation constrained multicast can be formulated as a constructive interference downlink channel with the set of precoders $\mathbf{h}_{1}^{H}, \ldots, \mathbf{h}_{K}^{H}$, where each one of these precoders is allocated a power of $\left|\nu_{k}\right|$ and associated with the symbol $d \times 1 \angle \nu_{k}$.

Corollary 3. The solution of problem $\mathrm{x}_{C I P M}$ with uniformly scaled SINR constraints is given simply by scaling the output vector of the original problem as follows:

$$
\mathbf{x}_{C I P M}(\mathbf{d}, \mathbf{H}, n \boldsymbol{\zeta})=\sqrt{n} \mathbf{x}_{C I P M}(\mathbf{d}, \mathbf{H}, \boldsymbol{\zeta})
$$

where $n \in \mathbb{R}^{+}$.

Proof. By definition, the normalized precoder $\hat{\mathbf{x}}_{C I P M}$ is to $\frac{\mathbf{x}_{C I P M}}{\left\|\mathbf{x}_{C I P M}\right\|}$. For any $\mathbf{x}_{C I P M}, \angle\left(\mathbf{h}_{k} \mathbf{x}_{C I P M}\right)=$ $\angle\left(\mathbf{h}_{k} \hat{\mathbf{x}}_{C I P M}\right), \forall k \in K$. Therefore, all users can receive their target data symbols $\mathbf{d}$ scaled to a certain SNR value. This 


$$
\begin{array}{ccc}
0.5 K\left\|\mathbf{h}_{1}\right\|\left(\sum_{k}\left(-\mu_{k}+\alpha_{k} i\right)\left\|\mathbf{h}_{k}\right\| \rho_{1 k}\right. & - & \left.\sum_{k}\left(-\mu_{k}+\alpha_{k} i\right)\left\|\mathbf{h}_{k}\right\| \rho_{1 k}^{*}\right)=\sqrt{\zeta_{1}} \mathcal{I}\left(d_{1}\right) \\
0.5 K\left\|\mathbf{h}_{1}\right\|\left(\sum_{k}\left(-\mu_{k} i-\alpha_{k}\right)\left\|\mathbf{h}_{k}\right\| \rho_{1 k}\right. & + & \left.\sum_{k}\left(-\mu_{k} i-\alpha_{k}\right)\left\|\mathbf{h}_{k}\right\| \rho_{1 k}^{*}\right)=\sqrt{\zeta_{1}} \mathcal{R}\left(d_{1}\right) \\
& \vdots & \\
0.5 K\left\|\mathbf{h}_{K}\right\|\left(\sum_{k}\left(-\mu_{k}+\alpha_{k} i\right)\left\|\mathbf{h}_{k}\right\| \rho_{K k}\right. & \left.-\sum_{k}\left(-\mu_{k}+\alpha_{k} i\right)\left\|\mathbf{h}_{k}\right\| \rho_{K k}^{*}\right)=\sqrt{\zeta_{K}} \mathcal{I}\left(d_{K}\right) \\
0.5 K\left\|\mathbf{h}_{K}\right\|\left(\sum_{k}\left(-\mu_{k} i-\alpha_{k}\right)\left\|\mathbf{h}_{k}\right\| \rho_{K k}\right. & + & \left.\sum_{k}\left(-\mu_{k} i-\alpha_{k}\right)\left\|\mathbf{h}_{k}\right\| \rho_{K k}^{*}\right)=\sqrt{\zeta_{K}} \mathcal{R}\left(d_{K}\right)
\end{array}
$$

implies that scaling uniformly all users' SNR targets does not change $\hat{\mathbf{x}}_{C I P M}$. Using the simultaneous set of equations (36), we can replace each $\zeta_{j}$ by $n \zeta_{j}$. This multiplies each value of $\mu_{j}, \alpha_{j}$ by $\sqrt{n}$. As a consequence, a scaling factor of $\sqrt{n}$ is multiplied with the original output vector $\mathbf{x}$ which proves the corollary.

\section{Constructive Interference Power Minimization Bounds}

In order to assess the performance of the proposed algorithm, we mention below two theoretical upper bounds.

1) Genie-aided upper-bound: This bound occurs when all multiuser transmissions are constructively interfering by nature and without the need to optimize the output vector. The minimum transmit power for a system that exploits the constructive interference on symbol basis can be found by the following theoretical bound

Theorem 2. The genie-aided minimum transmit power in the downlink of multiuser MISO system can be found by solving the following optimization

$\begin{aligned} P_{\text {min }} & =\arg \min _{p_{1}, \ldots, p_{K}} \sum_{k=1}^{K} p_{k} \\ \text { s.t. } & \left\|\mathbf{g}_{k}\right\|^{2}\left(\left|\xi_{k k}\right|^{2} p_{k}+\sum_{j=1, j \neq k}^{K} p_{j}\left|\xi_{k j}\right|^{2}\right) \geq \zeta_{k}, \forall k \in K .\end{aligned}$

Proof. According to (19), the bound in (37) can be found if all users face a constructive interference with respect to the multiuser transmissions of all other streams $\angle\left(\xi_{j k} d_{j}\right)=$ $\angle d_{k}, \forall k, \forall j$.

This bound can be mathematically found by solving the problem (37) using linear programming techniques [28].

2) Optimal Multicast: Based on theorem 2, a theoretical upper-bound can be characterized. This bound occurs if we drop the phase alignment constraint $\mathcal{C}_{1}$. The intuition of using this technique is the complete correlation among the information that needs to be communicated (i.e. same symbol for all users). The optimal input covariance for power minimization in multicast system can be found as a solution of the following optimization

$$
\min _{\mathbf{Q}: \mathbf{Q} \succeq 0} \operatorname{tr}(\mathbf{Q}) \quad \text { s.t. } \quad \mathbf{h}_{j} \mathbf{Q} \mathbf{h}_{j}^{H} \geq \zeta_{j} \quad, \forall j \in K .
$$

This problem is thoroughly solved in [14]. A tighter upperbound can be found by imposing a unit rank constraint on Q [15], to allow the comparison with the unit rank transmit power minimization constructive interference precoding

$\min _{\mathbf{Q}: \mathbf{Q} \succeq 0, \operatorname{Rank}(\mathbf{Q})=1} \operatorname{tr}(\mathbf{Q}) \quad$ s.t. $\quad \mathbf{h}_{j} \mathbf{Q h}_{j}^{H} \geq \zeta_{j} \quad, \forall j \in K$
Eq. (39) presents a tighter upper bound in comparison (38). It assumes a unit rank approximation of (38).

VII. WeIghted MAX Min SINR Algorithm FOR CONSTRuctive InTERfERENCE Precoding (CIMM)

The weighted max-min SINR beamforming aims at improving the relative fairness in the system by maximizing the worst user SINR. This problem has been studied in different frameworks such as multicast [14], and downlink transmissions [10]. In [14], the authors have solved this problem by finding the relation between the min-power problem and max-min problem and formulating both problem as convex optimization ones. On the other hand, the authors of [10] have solved the problem using the bisection technique. In this work, we exploit the constructive interference to enhance the user fairness in terms of weighted SNR. The challenging aspect is the additional constraints which guarantee the data have been detected correctly at the receivers. The constructive interference max-min problem can be formulated as

$$
\begin{aligned}
\mathbf{w}_{k}=\operatorname{maxmin}_{\mathbf{w}_{k}} & \left\{\frac{\left\|\mathbf{h}_{j} \sum_{k=1}^{K} \mathbf{w}_{k} d_{k}\right\|^{2}}{r_{j}}\right\}_{i=1}^{K} \\
\text { s.t. } & \left\{\begin{array}{l}
\mathcal{C} 1:\left\|\sum_{k=1}^{K} \mathbf{w}_{k} d_{k}\right\|^{2} \leq P \\
\mathcal{C} 2: \angle\left(\mathbf{h}_{j} \sum_{k=1}^{K} \mathbf{w}_{k} d_{k}\right)=\angle\left(d_{j}\right), \quad \forall j \in K .
\end{array}\right.
\end{aligned}
$$

where $r_{j}$ denotes the requested SNR target for the $i^{\text {th }}$ user. If we denote $\mathbf{q}=\sum_{j=1}^{K} \mathbf{w}_{j} d_{j}$, the previous optimization can be formulated as

$$
\begin{aligned}
\mathbf{q}(\mathbf{d}, \mathbf{H}, \mathbf{r})=\max _{\mathbf{q}} \min _{j} & \left\{\frac{\left\|\mathbf{h}_{j} \mathbf{q}\right\|^{2}}{r_{j}}\right\}_{i=1}^{K} \\
\text { s.t. } & \left\{\begin{array}{l}
\mathcal{C} 1:\|\mathbf{q}\|^{2} \leq P \\
\mathcal{C} 2: \angle\left(\mathbf{h}_{j} \mathbf{q}\right)=\angle\left(d_{j}\right), \quad \forall j \in K
\end{array}\right.
\end{aligned}
$$

where $\mathbf{r}$ is the vector that contains all the weights $r_{j}$. In the following, it is shown that the optimal output vector is a scaled version of the min-power solution in (23) [14]. The weighted maximum minimum SINR problem has been solved using bisection method over $t \in[0,1][10]$.

Lemma 3. The relationship between min-power and max-min problem can be described as $\mathbf{q}(\mathbf{d}, \mathbf{H}, \mathbf{r})=\mathbf{x}\left(\mathbf{d}, \mathbf{H}, t^{*} \mathbf{r}\right)$.

Proof. The problem (41) can be formulated

$$
\begin{array}{ll}
\max _{t, \mathbf{q}} & t \\
\text { s.t. } & \left\{\begin{array}{l}
\mathcal{C} 1:\|\mathbf{q}\|^{2} \leq P \\
\mathcal{C} 2: \frac{\mathbf{h}_{j} \mathbf{q}-\left(\mathbf{h}_{j} \mathbf{q}\right)^{H}}{i\left(\mathbf{h}_{j} \mathbf{q}+\left(\mathbf{h}_{j} \mathbf{q}\right)^{H}\right)}=\tan \left(\angle d_{j}\right), \forall j \in K \\
\mathcal{C} 3: \mathcal{R}\left\{d_{j}\right\} \cdot \mathcal{R}\left\{\mathbf{h}_{j} \mathbf{q}\right\} \geq 0, \forall j \in K \\
\mathcal{C} 4: \mathcal{I}\left\{d_{j}\right\} \cdot \mathcal{I}\left\{\mathbf{h}_{j} \mathbf{q}\right\} \geq 0, \forall j \in K \\
\mathcal{C} 5:\left\|\mathbf{h}_{j} \mathbf{q}\right\|^{2} \geq r_{j} t, \forall j \in K .
\end{array}\right.
\end{array}
$$


The optimal value of $t$ denoted by $t^{*}$ can be found by solving the min-power.

Thus, the max-min SINR solution is a scaled version of min power solution, which means that the system designer needs to find the optimal value of $t^{*}$ to solve the max-min problem. In the next section, we propose a simple method that can find this parameter influenced by the literature [10].

\section{B. Max-min SINR Constructive Interference Precoding}

In comparison with (40), we have additional $3 K$ constraints that limit the system performance. The problem can be formulated as

$$
\begin{array}{ll}
\max _{t, \mathbf{q}} & t \\
\text { s.t. } & \left\{\begin{array}{l}
\mathcal{C} 1:\|\mathbf{q}\|^{2}=P \\
\mathcal{C} 2: \frac{\mathbf{h}_{j} \mathbf{q}-\left(\mathbf{h}_{j} \mathbf{q}\right)^{H}}{i\left(\mathbf{h}_{j} \mathbf{q}+\left(\mathbf{h}_{j} \mathbf{q}\right)^{H}\right)}=\tan \left(\angle d_{j}\right), \forall j \in K \\
\mathcal{C} 3: \frac{\left(d+d^{*}\right)}{2} \cdot \frac{\mathbf{h}_{j} \mathbf{q}+\left(\mathbf{h}_{j} \mathbf{q}\right)^{H}}{2} \geq 0, \forall j \in K \\
\mathcal{C} 4: \frac{\left(d-d^{*}\right)}{2 i} \cdot \frac{\mathbf{h}_{j} \mathbf{q}-\left(\mathbf{h}_{j} \mathbf{q}\right)^{H}}{2 i} \geq 0, \forall j \in K \\
\mathcal{C} 5:\left\|\mathbf{h}_{j} \mathbf{q}\right\|^{2} \geq r_{j} t, \forall j \in K .
\end{array}\right.
\end{array}
$$

A solution for (43) can be found in the same fashion by using the bisection method as [10] and can be summarized as

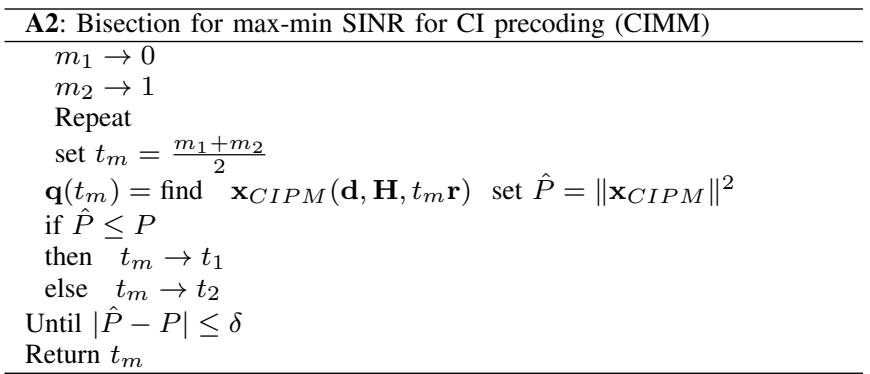

\section{Weighted Sum Rate MaXimization ALGORITHMS FOR CONSTRUCTIVE INTERFERENCE PRECODING (CISR)}

The sum rate problem of the multiuser downlink of multiple antennas for user-level precoding has been investigated in the literature [3]- [4]. The authors in [3] prove that the sum rate problem is NP hard. However, a simpler solution for the sum rate problem is characterized in [4] by rotating the MRTs of each user's channel to reduce the amount of the created interference on other users' transmissions. On the other hand, the weighted sum rate optimization in single group multicast scenarios is studied [16], which tries to design closed form precoders at different high SNR scenarios and proposes an iterative algorithm with low computation complexity for general SNR case. Furthermore, heuristic solutions for sum rate maximization of group multicast precoding with perantenna power constraint are proposed in [19].

In this work, we take into the account that the interference can be exploited among the different multiuser data streams. This requires that the sum rate problem should be formulated to take into consideration this new feature. The weighted sum rate maximization with a unit rank assumption for the precoding matrix can be written as ${ }^{3}$

$$
\begin{array}{ll}
\max _{\mathbf{q}} & \sum_{j=1}^{K} \phi_{j} \log _{2}\left(1+\frac{\left\|\mathbf{h}_{j} \mathbf{q}\right\|^{2}}{\sigma^{2}}\right) \\
\text { s.t. } & \left\{\begin{array}{l}
\mathcal{C}_{1}: \angle \mathbf{h}_{j} \mathbf{q}=\angle d_{j} \quad \forall j \in K . \\
\mathcal{C}_{2}:\|\mathbf{q}\|^{2} \leq P .
\end{array}\right.
\end{array}
$$

where $\phi_{j}$ is the weight related to the $j^{t h}$ user. The optimization can be formulated as

$$
\begin{array}{ll}
\max _{\mathbf{q}} & \sum_{j=1}^{K} \phi_{j} \log _{2}\left(1+\frac{\left\|\mathbf{h}_{j} \mathbf{q}\right\|^{2}}{\sigma^{2}}\right) \\
\text { s.t. } & \left\{\begin{array}{l}
\mathcal{C}_{1}: \mathcal{I}\left\{\mathbf{h}_{j} \mathbf{q}\right\}=i \tan \left(\angle d_{j}\right) \mathcal{R}\left\{\mathbf{h}_{j} \mathbf{q}\right\} \quad \forall j \in K, \\
\mathcal{C}_{2}:\|\mathbf{q}\|^{2} \leq P, \\
\mathcal{C}_{3}: \frac{\left(d_{j}+d_{j}^{*}\right)}{2} \cdot \frac{\mathbf{h}_{j} \mathbf{q}+\left(\mathbf{h}_{j} \mathbf{q}\right)^{H}}{2} \geq 0, \forall j \in K, \\
\mathcal{C}_{4}: \frac{\left(d_{j}-d_{j}^{*}\right)}{2 i} \cdot \frac{\mathbf{h}_{j} \mathbf{q}-\left(\mathbf{h}_{j} \mathbf{q}\right)^{H}}{2 i} \geq 0, \forall j \in K
\end{array}\right.
\end{array}
$$

\section{A. Modulation Selection}

In order to optimize the sum-rate, practical communication systems implement adaptive modulation and coding schemes (MCS) which adapt the density of the transmitted constellation to the current SNR. Unfortunately, this adaptation cannot be applied on a symbol-level because this would render the signalling overhead impractical. In this context, let us assume that the modulation of each user remains fixed during the channel coherence time of a quasi-static block fading channel. This way, each user has to be notified only once per $\tau_{c}$ about the constellation type that he has to detect. The modulation for each user is selected at the beginning of each coherence time. To decide the most appropriate modulation type for each user, we use the optimal multicast, which is defined as

$$
\mathbf{Q}_{o}=\arg \max _{\mathbf{Q}: t r(\mathbf{Q}) \leq P} \sum_{j=1}^{K} \phi_{j} \log _{2}\left(1+\frac{\mathbf{h}_{j} \mathbf{Q h}_{j}^{H}}{\sigma^{2}}\right),
$$

to decide the highest modulation order for each user for the whole transmission frame (i.e. here is assumed to be equal to the channel coherence time) by the following criteria

$$
\mathrm{MCS}=\left\{\begin{array}{l}
\text { no service, } \omega_{j}^{\prime} \leq \omega_{B P S K} \\
\text { BPSK, } \omega_{B P S K} \leq \omega_{j}^{\prime} \leq \omega_{Q P S K} \\
\vdots
\end{array}\right.
$$

where $\omega_{j}^{\prime}$ is the effective SNR at each user receiver. Due to the fact that M-PSK data symbols are encoded using the phase information, any higher order PSK symbol can be decoded as lower PSK if each user knows its target modulation. For example, the symbol $1 \angle 45^{\circ}=\frac{1+i}{2}$ can be detected as 11 if the agreed modulation between the transmitter and receiver is QPSK, and it can be detected as 1 if the agreed modulation is BPSK. As a result, when designing sum-rate CI algorithms at

\footnotetext{
${ }^{3}$ For the sum rate problem, it should be noted that the optimal solution is not necessarily unit rank, but we employ this assumption to enable tractable heuristic solutions.
} 
the transmitter, we can always assume that all users expect the highest-order PSK modulation. At the receiver, the demapping of the received symbol will depend on the assigned MCS.

\section{B. Genie-aided Sum Rate Upper Bound}

Based on theorem (2), the maximum sum rate can be found by solving the following optimization

$$
\begin{array}{ll}
\max _{p_{1}, \ldots, p_{K}} & \sum_{k=1}^{K} \log _{2}\left(1+\left\|\mathbf{g}_{k}\right\|^{2}\left(\left|\xi_{k k}\right|^{2} p_{k}+\sum_{j=1, j \neq k}^{K} p_{j}\left|\xi_{k j}\right|^{2}\right)\right) \\
\text { s.t. } & \sum_{k=1}^{K} p_{k} \leq P
\end{array}
$$

\section{Optimal solution}

The optimal solution cannot be found in a straight forward manner due to the different types of the constraints: the phase constraints and the threshold constraints. However, we write the Lagrangian function of the previous optimization (45) to get more insights about the problem as (49) and the derivative of the related sum rate problem (50). Moreover, it can be seen that it has different solutions than the power minimization problem.

1) Low SNR approximation: To simplify the analysis, we use the low SINR approximation $\log _{2}\left(1+\frac{\alpha_{i}}{\sigma^{2}}\right) \sim \frac{\alpha_{i}}{\sigma^{2}}$ which is valid in the regime $\sigma^{2} \rightarrow \infty$. Thus the optimization problem (45) can be written as

$$
\begin{array}{ll}
\max _{\mathbf{q}} & \sum_{j=1}^{K} \phi_{j} \frac{\left\|\mathbf{h}_{j} \mathbf{q}\right\|^{2}}{\sigma^{2}} \\
\text { s.t. } & \left\{\begin{array}{l}
\mathcal{C}_{1}: \frac{\mathbf{h}_{j} \mathbf{q}-\mathbf{q}^{H} \mathbf{h}_{j}^{H}}{\left(\mathbf{h}_{j} \mathbf{q}+\mathbf{q}^{H} \mathbf{h}_{j}^{H}\right)}=i \tan \left(\angle d_{j}\right) \quad \forall j \in K, \\
\mathcal{C}_{2}:\|\mathbf{q}\|^{2} \leq P, \\
\mathcal{C}_{3}: \frac{\left(d_{j}+d_{j}^{*}\right)}{2} \frac{\mathbf{h}_{j} \mathbf{q}+\left(\mathbf{h}_{j} \mathbf{q}\right)^{H}}{2} \geq 0, \forall j \in K, \\
\mathcal{C}_{4}: \frac{\left(d_{j}-d_{j}^{*}\right)}{2 i} \frac{\mathbf{h}_{j} \mathbf{q}-\left(\mathbf{h}_{j} \mathbf{q}\right)^{H}}{2 i} \geq 0, \forall j \in K
\end{array}\right.
\end{array}
$$

and the corresponding Lagrangian function can be written as

$$
\begin{aligned}
\mathcal{L}(\mathbf{q}) & =\sum_{j=1}^{K} \phi_{j} \frac{\left\|\mathbf{h}_{j} \mathbf{q}\right\|^{2}}{\sigma^{2}}+\sum_{j=1}^{K} \pi_{j}\left(\mathbf{h}_{j} \mathbf{q}-\mathbf{q}^{H} \mathbf{h}_{j}^{H}-i \tan \left(\angle d_{j}\right)\right. \\
& \left.\times\left(\mathbf{h}_{j} \mathbf{q}+\mathbf{q}^{H} \mathbf{h}_{j}^{H}\right)\right)+\sum_{j=1}^{K} \beta_{j} \frac{\left(d_{j}+d_{j}^{*}\right)}{2} \frac{\mathbf{h}_{j} \mathbf{q}+\left(\mathbf{h}_{j} \mathbf{q}\right)^{H}}{2} \\
& +\sum_{j=1}^{K} \alpha_{j} \frac{\left(d_{j}-d_{j}^{*}\right)}{2 i} \frac{\mathbf{h}_{j} \mathbf{q}-\left(\mathbf{h}_{j} \mathbf{q}\right)^{H}}{2 i}+\psi\left(\mathbf{q}^{H} \mathbf{q}-P\right) .
\end{aligned}
$$

If we denote $\alpha_{j}^{\prime}=\alpha_{j} \frac{d_{j}-d_{j}^{*}}{2 i}, \beta_{j}^{\prime}=\beta_{j} \frac{d_{j}+d_{j}^{*}}{2}, \pi_{j}^{\prime}=-\pi_{j}(1+$ $\left.i \tan \left(\angle d_{j}\right)\right)$, the derivative of (52) can be formulated as

$$
\frac{d \mathcal{L}(\mathbf{q})}{d \mathbf{q}^{*}}=\sum_{j=1}^{K} \phi_{j} \frac{\mathbf{h}_{j}^{H} \mathbf{h}_{j} \mathbf{q}}{\sigma^{2}}+\sum_{j=1}^{K}\left(\alpha_{j}^{\prime}+\pi_{j}^{\prime}+\beta_{j}^{\prime}\right) \mathbf{h}_{j}^{H}+\psi \mathbf{q} .
$$

Then, $\mathbf{q}$ can be expressed as

$$
\mathbf{q}=\left(\sum_{j=1}^{K} \phi_{j} \frac{\mathbf{h}_{j}^{H} \mathbf{h}_{j}}{\sigma^{2}}+\psi \mathbf{I}\right)^{-1}\left(\sum_{j=1}^{K}\left(\alpha_{j}^{\prime}+\pi_{j}^{\prime}+\beta_{j}^{\prime}\right) \mathbf{h}_{j}^{H}\right)
$$

Since the assumed approximation works in the low SNR regime (i.e. noise limited scenario $\sigma^{2} \rightarrow \infty$ ), the expression in (54) can be simplified into the following expression

$$
\mathbf{q}=\sum_{j=1}^{K} \underbrace{\left(\pi_{j}^{\prime}+\beta_{j}^{\prime}+\alpha_{j}^{\prime}\right)}_{a_{j}} \mathbf{h}_{j}^{H}
$$

It can be noted that the precoding formulation at the low SNR regime resembles the generic formula of min power precoding. Moreover, the weight for each user vanishes in this regime. Based on this fact, we propose heuristic precoding schemes that aim at maximizing the sum rate of the downlink multiuser transmissions.

\section{Heuristic schemes}

Since the solution for the sum rate maximization problem in (44) is difficult to find, we propose two heuristic algorithms to tackle this problem as follows

1) Phase alignment algorithm: The sum rate maximization problem can be solved exploiting the low SNR approximation expression in (55). This expression contains $3 K$ variables (i.e. $\alpha_{j}^{\prime}, \beta_{j}^{\prime}$, and $\pi_{j}^{\prime} \forall j \in K$ ) that have to satisfy the phase alignment constraints $\mathcal{C}_{1}$ in (44) while it should maximize the sum rate in the system. Utilizing the eigenvectors of $\mathbf{H H}^{H}$, q can be formulated as

$$
\mathbf{q}=\sum_{j=1}^{K} a_{j} \mathbf{h}_{j}^{H}=\sum_{j=1}^{K} b_{j} \mathbf{e}_{j}
$$

where $\mathbf{e}_{j}$ is the $\mathrm{j}^{\text {th }}$ eigenvector of $\mathbf{H} \mathbf{H}^{H}$, and $b_{j} \in \mathbb{C}^{1 \times 1}$ is the weight associated with $j^{t h}$ eigenvector. This makes the received SINR formulated as

$$
\gamma_{j}=\sum_{i=1}^{K}\left|b_{i}\right|^{2} \mathbf{h}_{j} \mathbf{e}_{i} \mathbf{e}_{i}^{H} \mathbf{h}_{j}^{H}
$$

Using (56), the optimization (45) can be reformulated as

$$
\begin{array}{ll}
\max _{b_{i}} & \sum_{j=1}^{K} \log _{2}\left(1+\gamma_{j}\right) \\
\text { s.t. } & \left\{\begin{array}{l}
\mathcal{C}_{1}: \angle \mathbf{h}_{j}\left(\sum_{i=1}^{K} b_{i} \mathbf{e}_{i}\right)=\angle d_{j}, \forall j \in K \\
\mathcal{C}_{2}: \sum_{i=1}^{K}\left|b_{i}\right|^{2} \leq P .
\end{array}\right.
\end{array}
$$

\section{A3: Sum Rate Maximization - Phase alignment algorithm (CISR-PA)}

1) Solve the optimization (57) without $\mathcal{C}_{2}$ and find $\left|b_{i}\right|$.

2) Select the modulation type for each user based on the achieved SINR $\zeta_{j}$.

3) Solve the following set of equations by finding $\angle b_{i}$

$$
\mathbf{h}_{j}\left(\sum_{i=1}^{K}\left|b_{i}\right| \mathbf{e}_{i} \exp \left(\angle b_{i}\right) 1 i\right)=\sqrt{\zeta_{j}} 1 \angle d_{j}, \forall j \in K
$$

This set of equations is required to assure that the phase constraints $\mathcal{C}_{1}$ in (57) are satisfied.

4) Scale $\mathbf{q}$ by setting $\|\mathbf{q}\|^{2}=P$ 


$$
\begin{aligned}
\mathcal{L}(\mathbf{q}) & =\sum_{j=1}^{K} \phi_{j} \log _{2}\left(1+\frac{\left\|\mathbf{h}_{j} \mathbf{q}\right\|^{2}}{\sigma^{2}}\right)+\sum_{j=1}^{K} \mu_{j}\left(\frac{\left(d_{j}+d_{j}^{*}\right)}{2} \frac{\mathbf{h}_{j} \mathbf{q}+\left(\mathbf{h}_{j} \mathbf{q}\right)^{H}}{2}\right)+\sum_{j=1}^{K} \alpha_{j}\left(\frac{\left(d_{j}-d_{j}^{*}\right)}{2 i} \frac{\mathbf{h}_{j} \mathbf{q}-\left(\mathbf{h}_{j} \mathbf{q}\right)^{H}}{2 i}\right) \\
& +\sum_{j=1}^{K} \kappa_{j}\left(\mathbf{h}_{j} \mathbf{q}-\mathbf{q}^{H} \mathbf{h}_{j}^{H}-i \tan \left(\angle d_{j}\right)\left(\mathbf{h}_{j} \mathbf{q}+\mathbf{q}^{H} \mathbf{h}_{j}^{H}\right)\right)+\gamma\left(\mathbf{q}^{H} \mathbf{q}-P\right) \\
\frac{d \mathcal{L}(\mathbf{q})}{d \mathbf{q}^{*}}= & \sum_{j=1}^{K} \phi_{j} \frac{\mathbf{h}_{j}^{H} \mathbf{h}_{j} \mathbf{q}}{\sigma^{2}+\mathbf{q}^{H} \mathbf{h}_{j}^{H} \mathbf{h}_{j} \mathbf{q}}+\sum_{j=1}^{K} \mu_{j} \frac{d_{j}+d_{j}^{*}}{4} \mathbf{h}_{j}^{H}+\sum_{j=1}^{K} \alpha_{j} \frac{\left(d_{j}-d_{j}^{*}\right)}{2} \mathbf{h}_{j}^{H}-\sum_{j=1}^{K} \kappa_{j}\left(1+i \tan \left(\angle d_{j}\right)\right) \mathbf{h}_{j}^{H}+\gamma \mathbf{q}(50)
\end{aligned}
$$

2) Greedy Algorithm: In this algorithm, we jointly utilize the solution for the unconstrained optimal sum rate maximization multicast problem (46) and the constrained constellation power minimization problem (23) to propose a new heuristic algorithm. The intuition of using such algorithm is to find the subset of users that has similar characteristics in terms of the co-linearity with respect to the optimal multicast directions (i.e. the projection to eigenvector associated with the maximum eigenvalue of $\mathbf{Q}_{o}$ ). It should be noted that this co-linearity is defined by the projection and the angle of the projection as it is illustrated in the following algorithm

A4: Greedy sum rate maximization (CISR-G)

1) Find the optimal input covariance by solving the unconstrained multicast problem (46).

2) Find the optimal direction (i.e. the maximum eigenvector $\mathbf{Q}_{\circ}$ ) that maximizes the projection

$$
\boldsymbol{\Phi}_{o}=\arg \max _{\mathbf{\Phi}} \quad \mathbf{\Phi} \mathbf{Q}_{o} \boldsymbol{\Phi}^{H}
$$

3) For all $j$, evaluate $\mathbf{g}_{j}=\mathbf{h}_{j} \boldsymbol{\Phi}_{\circ}$.

4) Find $j^{*}=\arg \max _{j}\left\|\mathbf{g}_{j}\right\|^{2}$

5) Select the modulation order based on (47), using $\omega_{j}^{\prime}$

6) For all possible combinations $\mathcal{G}=\cup_{j} \mathcal{K}_{j}$, evaluate the sum of the users' projection and

$$
\Lambda(\mathcal{K})=\sum_{j \in \mathcal{K}_{j} \subset \mathcal{G}} \mathbf{g}_{j} .
$$

7) Select the subset of users for all possible of users combinations and find the maximum that has the highest projection

$$
\mathcal{K}_{j}^{*}=\arg \max _{\mathcal{K}_{j}}\left\|\Lambda\left(\mathcal{K}_{j}\right)\right\|^{2}
$$

8) Evaluate the respective power minimization problem SNR target values.

$$
\begin{gathered}
\iota_{j}=\frac{\log _{2}\left(\left\|\mathbf{g}_{j}\right\|^{2}\right)}{\sum_{i \in \mathcal{K}_{j}^{*}} \log _{2}\left(\left\|\mathbf{g}_{i}\right\|^{2}\right)} \\
\zeta_{j}^{\prime}=\left\{\begin{array}{l}
\left\|\mathbf{g}_{j}\right\|^{2}, \text { if }\left\|\mathbf{g}_{j}\right\|^{2} \leq \iota_{j}\left\|\mathbf{g}_{j}^{*}\right\|^{2} \\
\iota_{j}\left\|\mathbf{g}_{j}^{*}\right\|^{2}, \text { if }\left\|\mathbf{g}_{j}\right\|^{2} \geq \iota_{j}\left\|\mathbf{g}_{j}^{*}\right\|^{2}
\end{array}\right.
\end{gathered}
$$

9) Solve the related power minimization problem

$$
\begin{array}{ll}
\min _{\mathbf{q}} & \mathbf{q}^{H} \mathbf{q} \\
\text { s.t. } & \left\{\begin{array}{l}
\mathcal{C} 1: \frac{\mathbf{h}_{i} \mathbf{q}-\left(\mathbf{h}_{i} \mathbf{q}\right)^{H}}{2 i}=\sigma \sqrt{\zeta_{j}^{\prime}} \mathcal{I}\left\{d_{i}\right\}, \forall i \in \mathcal{K}_{i}^{*} \\
\mathcal{C} 2: \frac{\mathbf{h}_{i} \mathbf{q}+\left(\mathbf{h}_{i} \mathbf{q}\right)^{H}}{2}=\sigma \sqrt{\zeta_{i}^{\prime}} \mathcal{R}\left\{d_{i}\right\}, \forall i \in \mathcal{K}_{i}^{*} .
\end{array}\right.
\end{array}
$$

10) Then, scale $\mathbf{q}$ such that $\|\mathbf{q}\|^{2}=P$.

\section{Algorithms COMPLEXITY}

The complexity of the proposed algorithms is an important aspect to assess their feasibility. A discussion about the complexity of each algorithms can be summarized as follows:

- CIMRT requires an SVD to be employed on the channel $\mathbf{H}$ which has the complexity of $4 M^{2} K+8 M K^{2}+9 K^{3}$ and to solve $\frac{K^{2}-K}{2}$ times a set of two non-linear equations simultaneously.

- CIPM requires solving $2 K$ linear equations simultaneously, which means that it has less complexity than CIMRT

- CIMM requires solving $2 K$ linear equations simultaneously at each bisection iteration. Moreover, the bisection has a complexity of $\log _{2}\left(\max r_{k}\right)$.

- CISR-PA requires solving the optimization in (57) without $\mathcal{C}_{2}$ which calls numerical solvers such as SeDuMi [14]. In order to solve this convex optimization, we need to find the eigenvalue decomposition of $\mathbf{H H}^{H}$ which has the complexity of $4 M^{2} K+(8 M+1) K^{2}+9 K^{3}$. Moreover, this algorithm requires solving $K$ linear equations simultaneously.

- CISR-G requires solving the convex optimization (46) without imposing any rank constraint on $\mathbf{Q}$ and assuming it is a positive semidefinite, and finding the eigenvector associated with maximum eigenvalue. Furthermore, it needs to search all possible combinations $\sum_{i}^{K}\left(\begin{array}{c}K \\ i\end{array}\right)$ to select the most suitable subset of users to serve in coherence time. Finally, we need to solve $2 K$ linear equations simultaneously.

\section{Numerical Results}

In order to assess the performance of the proposed transmissions schemes, Monte-Carlo simulations of the different algorithms have been conducted to study the performance of the proposed techniques and compare to the state of the art techniques. The adopted channel model is assumed to be

$$
\mathbf{h}_{k} \sim \mathcal{C N}\left(0, \sigma_{c}^{2}\right) .
$$

We define the energy efficiency metric as follows

$$
\eta=\sum_{j=1}^{K} \frac{R_{j}}{P},
$$

where $R_{j}=\log _{2}\left(1+\omega_{j}\right)$. 


\begin{tabular}{|c|c|c|}
\hline Acronym & Technique & $\begin{array}{l}\text { equation or } \\
\text { algorithm }\end{array}$ \\
\hline CIZF & Constructive Interference Zero Forcing & (12) \\
\hline CIMRT & $\begin{array}{l}\text { Constructive Interference Maximum Ratio } \\
\text { Transmissions }\end{array}$ & (21), A1 \\
\hline CIPM & $\begin{array}{l}\text { Constructive Interference- Power Mini- } \\
\text { mization }\end{array}$ & (35) \\
\hline CIMM & $\begin{array}{l}\text { Constructive Interference-Maximization } \\
\text { the minimum SINR }\end{array}$ & A2 \\
\hline CISR-G & $\begin{array}{l}\text { Constructive Interference-Sum Rate max- } \\
\text { imization with Greedy approach }\end{array}$ & $\mathbf{A 3}$ \\
\hline CISR-PA & $\begin{array}{l}\text { Constructive Interference-Sum Rate max- } \\
\text { imization with phase alignment }\end{array}$ & A4 \\
\hline GE & Genie aided upperbound & (37) \\
\hline Multicast & Optimal Multicast & (38) \\
\hline
\end{tabular}

SUMMARY OF THE PROPOSED ALGORITHMS, THEIR RELATED ACRONYMS, AND THEIR RELATED EQUATIONS AND ALGORITHMS

The motivation of using this metric is the fact that CRZF and CIMRT are achievable constructive techniques and cannot be designed based on optimization problems.

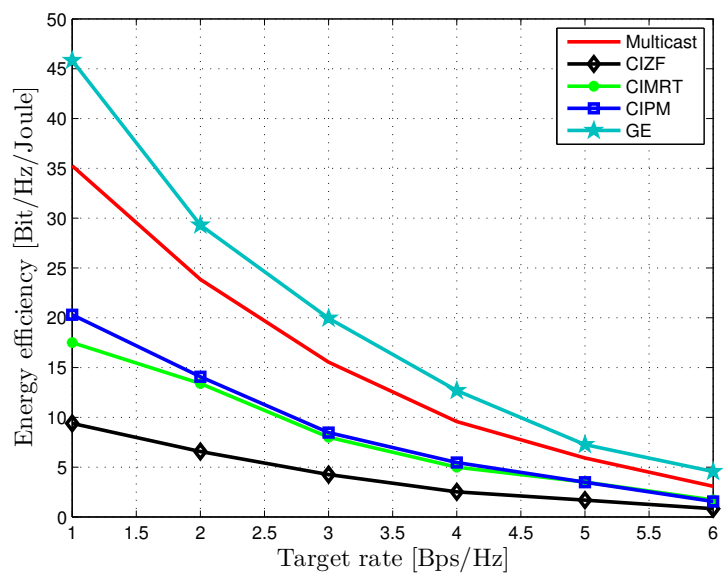

Fig. 3. Energy efficiency vs target rate.

In Fig. (3), we depict the performance of the proposed techniques from energy efficiency perspective with respect to the target SNR. To address this issue, the transmit power of the CIZF and CIMRT solutions can be scaled until all users achieve the target rate. We assume the scenario of $M=3$, $K=2$. Since there are no phase alignment constraints in the unconstrained multicast, it is anticipated that optimal multicast achieves a higher energy efficiency than CIPM and this is confirmed by simulations. Moreover, the gap between the genie-aided theoretical bound and multicasting reduces with increasing the target rate (i.e. modulation order for the genieaided). On the contrary, CIZF shows inferior performance in comparison with all depicted techniques. It has already been proven that CIZF outperforms the conventional techniques like minimum mean square error (MMSE) beamforming and zero forcing beamforming (ZFB) [23]. In comparison with other depicted techniques, it can be concluded that the proposed constructive interference with the CIPM achieves better energy efficiency in comparison with CIZF. This can be explained by the channel inversion step in CIZF which wastes energy in decoupling the effective users' channels and then exploit the interference among the multiuser streams. Moreover, it can be deduced that CIMRT has a very close performance to CIPM especially at high targets. CIMRT outperforms CIZF at expense of complexity. It can be concluded that at low target rates CIMRT has a different performance, CIMRT demonstrates increased variance among the achieved rates of each user. This fact in combination with the power scaling explains the CIMRT behavior for low target rates.

The comparison among the optimal multicast, the CIPM and the genie-aided bound is illustrated in Fig. (4). The assumed scenario $K=2, M=2$. It can be concluded that the power consumption gap between the optimal multicast and CIPM is fixed for all target rates. This relation holds also for the gap between the genie-aided upper-bound and CIPM.

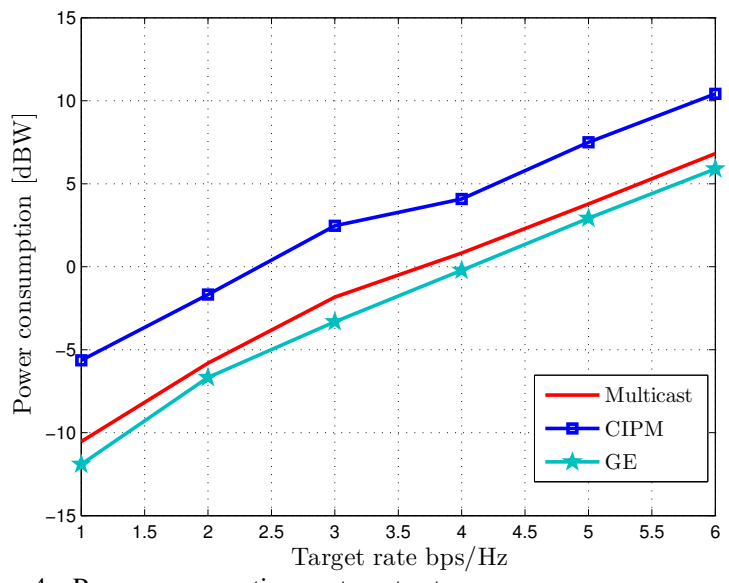

Fig. 4. Power consumption vs target rate.

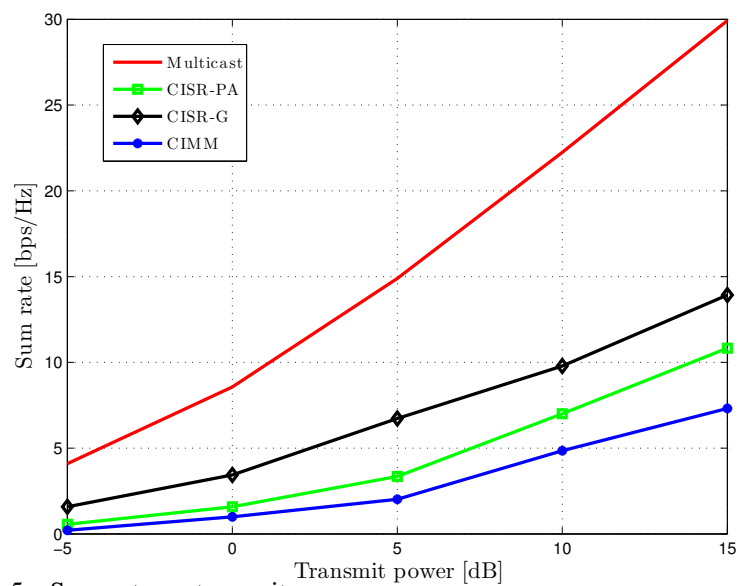

Fig. 5. Sum rate vs transmit power.

The sum rate performance is illustrated in Fig. (5) in the low-mid SNR regime. In this scenario, we consider $K=5$, $M=5$ with equal weights for the sum rate and max-min problems. It can be noted that the sum rate of algorithm 3, which is labeled in figure as CISR-G outperforms the phase alignment algorithm. It can be concluded that at low SNR, it is better to pre-select the users that have suitable channels to work together. In the constructive interference scenario, we tend to select the users whose channels are co-linear 


\begin{tabular}{|l|l|l|l|l|}
\hline$(\mathrm{M}, \mathrm{K})$ & $(2,2)$ & $(3,3)$ & $(4,4)$ & $(5,5)$ \\
\hline CIZF & 3.1220 & 1.9899 & 1.4248 & 1.2253 \\
\hline CIPM & 3.6159 & 3.1138 & 2.9871 & 2.8263 \\
\hline
\end{tabular}

TABLE II

ENERGY EFFICIENCY AT DIFFERENT $(\mathrm{M}, \mathrm{K})$ SCENARIOS

which opposes the conventional multiuser MISO techniques. However, for the same scenario, in the high SNR regime which is depicted in Fig. (6), the phase alignment algorithm CISR-PA shows a better performance than CISR-G, this means that it is better not to pre-select the users and serve all $K$ users. The performance gap between the two algorithms increases with SNR. The resulted loss of finding all the phases in CISR-PA has less effect on the system performance in comparison to switching off a few users. One should keep in mind that in all scenarios multicast is just an upper bound and is incapable of delivering different messages to different user. The difference in power consumption is anticipated since the sum rate problem does not take into the account the user with the weakest SNR. Finally, it can be concluded that the sum rate for the fairness achieving algorithm CIMM is less than CISR-G and CISR-PA in the low SNR regime. While for high SNR, this fact changes CIMM performs better than CISR-G and worse than CISR-PA.

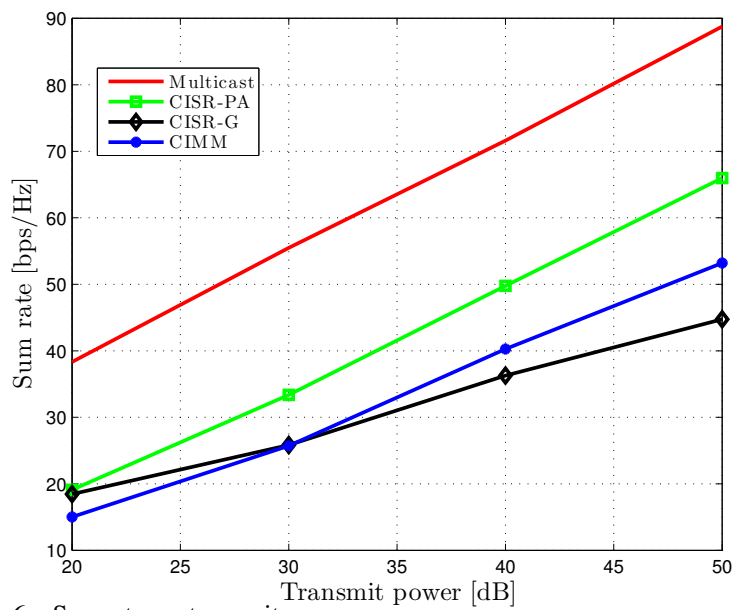

Fig. 6. Sumrate vs transmit power.

Table II discusses the performance of CIZF and CIPM in terms of energy efficiency with respect to $(M, K)$. It can be noted that energy efficiency decreases with increasing $(M, K)$. Moreover, the energy efficiency decreases more rapidly in the scenario of CIZF. This trend holds as $(M, K)$ increases.

\section{CONCLUSIONS}

In this paper, we exploit the CSI and DI to constructively correlate the transmitted symbols in symbol-level precoding. This enables interference exploitation among the multiuser transmissions assuming M-PSK modulation. Based on the idea of correlating the transmitted vectors, the connection between the constructive interference precoding and multicast precoding is characterized. We present several constructive interference designs from different perspective: minimizing the transmitted power while guaranteeing certain SNR thresholds for all users, maximizing the fairness among the users, and boosting the sum rate with fixed transmit power. From the results, it can be concluded that the max-min SINR problem is related to the power minimization problem. Moreover, we tackle the sum rate maximization problem and propose heuristic solutions to solve the problem. From the simulations, it can be concluded that the CIPM has a fixed transmit power gap with respect to multicast at different target rates. The sum rate maximization heuristic algorithms vary according to the SNR; CISR-G works very well at low SNR and this changes at high SNR while CISR-PA performs well at high SNR and this pattern changes at low SNR. Constructive interference techniques for M-QAM modulation will be presented in future work.

\section{REFERENCES}

[1] R. H. Roy and B. Ottersten, " Spatial division multiple access wireless communication systems, US patent, 1991.

[2] D. Gesbert, M. Kountouris, R. W. Heath Jr., C.-B. Chae and T. Sälzer,“ From Single User to Multiuser Communications: Shifting the MIMO Paradigm," IEEE Signal Processing Magazine, vol. 24 no.5, pp. 36-46, 2007.

[3] Y.-F. Liu, Y.-H. Dai, and Z.-Q. Luo, "Coordinated beamforming for MISO interference channel: Complexity analysis and efficient algorithms,"IEEE Transactions on Signal Processing, vol. 59, no. 3, pp. 1142-1157, 2011.

[4] E. Björnson, M. Bengtsson and B. Ottersten, "Optimal Multi-User Transmit Beamforming: Difficult Problem with a Simple Solution Structure," IEEE Signal Processing Magazine, vol. 31, no. 4, pp. 142-148, 2014.

[5] A. B. Gershman, N. D. Sidiropoulos, S. ShahbazPanahi, M. Bengtsson, and B. Ottersten, "Convex Optimization Based Beamforming," IEEE Signal Processing Magazine, vol. 27, no. 3, pp. 62-75, May 2010.

[6] M. Bengtsson and B. Ottersten,"Optimal and Suboptimal Transmit beamforming," in Handbook of Antennas in Wireless Communications, L. C. Godara, Ed. CRC Press, 2001.

[7] M. Schubert and H. Boche, "Solution of the Multiuser Downlink Beamforming Problem with Individual SINR Constraints," IEEE Transaction on Vehicular Technology, vol. 53, pp. 1828, January 2004.

[8] G. Caire, and S. Shamai (Shitz), "On the Achievable Throughput of a Multiantenna Gaussian Broadcast Channel," IEEE Transactions on Information Theory, vol. 49, no. 7, pp. 1691 - 1706, July 2002.

[9] Q. H. Spencer, A.L. Swindlehurst, and M. Haardt, "Zero-forcing Methods for Downlink Spatial Multiplexing in Multiuser MIMO Channels,"IEEE Transactions on Signal Processing, vol. 52, no.2, pp. 461-471, February 2004.

[10] A. Wiesel, Y. C. Eldar, and S. Shamai, "Linear precoding via conic optimization for fixed MIMO receivers," IEEE Transactions on Signal Processing, vol. 54, no. 1, pp. 161-176, 2006.

[11] Y. Wu, M. Wang, C. Xiao, Z. Ding and X. Gao, "Linear Precoding for MIMO Broadcast Channels with Finite-Alphabets Constraints," IEEE Transactions on Wireless Communications, vol. 11, no. 8, pp. 2906-2920, August 2012.

[12] H. Boche, M. Schubert, "Resource allocation in multiantenna systems-achieving max-min fairness by optimizing a sum of inverse SIR," IEEE Transactions on Signal Processing, vol. 54 no. 6, pp. 1990-1997, 2006.

[13] R. Ghaffar and R. Knopp, "Near Optimal Linear Precoding for Multiuser MIMO for Discrete Alphabets," IEEE International Conference on Communications (ICC), pp. 1-5, May 2010

[14] N. D. Sidropoulos, T. N. Davidson, ans Z.-Q. Luo, "Transmit Beamforming for Physical-Layer Multicasting," IEEE Transactions on Signal Processing, vol. 54, no. 6, pp. 2239-2251, June 2006.

[15] N. Jindal and Z.-Q. Luo, "Capacity Limits of Multiple Antenna Multicast," IEEE International Symposium on Information Theory (ISIT), pp. 1841 - 1845, June 2006.

[16] B. Du, M. Chen, W. Zhang and C. Pan, "Optimal beamforming for single group multicast systems based on weighted sum rate," IEEE International Conference on Communications(ICC), pp. 4921- 4925, June 2013.

[17] E. Jorswieck, "Beamforming in Interference Networks: Multicast, MISO IFC and Secrecy Capacity," International Zurich Seminars (IZS), March 2011.

[18] E. Karipidis, N. Sidiropoulos and Z.-Q Luo, "Transmit Beamforming to multiple Co-channel Multicast Groups," IEEE International Workshop on Computational Advances in Multi-Sensor Adaptive Processing (CAMSAP), pp. 109-112, December 2005. 
[19] D. Christopoulos, S. Chatzinotas and B. Ottersten, "Weighted Fair Multicast Multigroup Beamforming under Per-antenna Power Constraints," IEEE Transactions on Signal Processing, vo. 62, no. 19, pp. 5132-5142, 2014. Available on arXiv:1406.7557 [cs.IT].

[20] D. Christopoulos, S. Chatzinotas and B. Ottersten, "Multicast Multogroup Precoding and User Scheduling for Frame-based Satellite Communications," Submitted to IEEE Transactions on Wireless Communications, available arXiv:1406.7699 [cs.IT], 2014

[21] Y. C. B. Silva and A. Klein, "Linear Transmit Beamforming Techniques for the Multigroup Multicast Scenario,'IEEE Transaction on Vehicular Technology, vol. 58 , no. 8, pp. 4353 - 4367, October 2009.

[22] C. Masouros and E. Alsusa, "Dynamic Linear Precoding for the exploitation of Known Interference in MIMO Broadcast Systems," IEEE Transactions On Communications, vol. 8, no. 3, pp. 1396 - 1404, March 2009.

[23] C. Masouros, "Correlation Rotation Linear Precoding for MIMO Broadcast Communications," IEEE Transactions on Signal Processing, vol. 59, no. 1, pp. 252 262, January 2011.

[24] C. Masouros and T. Ratnarajah, "Interference as a source of green signal power in cognitive relay assisted co-existing MIMO wireless transmissions," IEEE Transactions on Communications, vol. 60, no. 2, pp. 525 - 536, February 2012.

[25] C. Masouros M. Sellathurai, and T. Ratnarajah, "Interference Optimization for Transmit Power Reduction in Tomlinson-Harashima Precoded MIMO Downlinks," IEEE Transactions on Signal Processing, vol. 60, no. 5, pp. 2470-2481, May 2012.

[26] M. Alodeh, S. Chatzinotas and B. Ottersten, "Data Aware User Selection in the Cognitive Downlink MISO Precoding Systems," invited paper to IEEE International Symposium on Signal Processing and Information Technology (ISSPIT), December 2013.

[27] M. Alodeh, S. Chatzinotas and B. Ottersten, "A Multicast Approach for Constructive Interference Precoding in MISO Downlink Channel," International Symposium in Information theory (ISIT) 2014, Available on arXiv:1401.6580v2 [cs.IT].

[28] S. Boyd, and L. Vandenberghe, Convex Optimization, Cambridge University press.

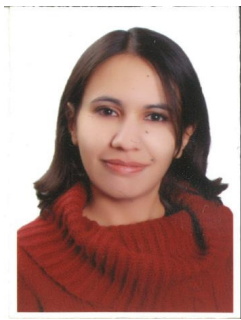

Maha Alodeh (S'11) received her bachelor degree in electrical engineering from University of Jordan, Amman, Jordan in 2010, and she is currently pursuing her Ph.D. degree in electrical engineering from the Interdisciplinary center for Security and Trust, (SnT), University of Luxembourg. Alodeh's research interests includes signal processing for wireless and satellite communications with focus on interference management and cognitive radios.

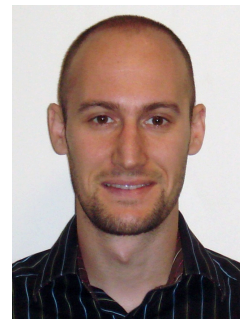

Symeon Chatzinotas (S'06-M'09-SM'13) ) received the M.Eng. in Telecommunications from Aristotle University of Thessaloniki, Greece and the M.Sc. and Ph.D. in Electronic Engineering from University of Surrey, UK in 2003, 2006 and 2009 respectively. He is currently a Research Scientist with the research group SIGCOM in the Interdisciplinary Centre for Security, Reliability and Trust, University of Luxembourg, managing H2020, ESA and FNR projects. In the past, he has worked in numerous R\&D projects for the Institute of Informatics \& Telecommunications, National Center for Scientific Research Demokritos, the Institute of Telematics and Informatics, Center of Research and Technology Hellas and Mobile Communications Research Group, Center of Communication Systems Research, University of Surrey. He has authored more than 120 technical papers in refereed international journals, conferences and scientific books. His research interests are on multiuser information theory, cooperative/cognitive communications and wireless networks optimization. Dr Chatzinotas is the co-recipient of the 2014 Distinguished Contributions to Satellite Communications Award, Satellite and Space Communications Technical Committee, IEEE Communications Society. He is currently coediting a book on "Cooperative and Cognitive Satellite Systems" to appear in 2015 by Elsevier and he is co-organizing the First International Workshop on Cognitive Radios and Networks for Spectrum Coexistence of Satellite and Terrestrial Systems (CogRaN-Sat) in conjunction with the IEEE ICC 2015, 8-12 June 2015, London, UK.

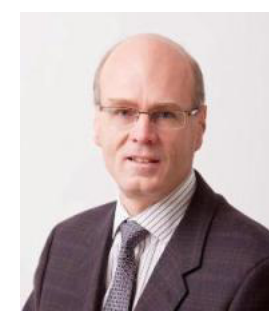

Björn Ottesten (S'87-M'89-SM'99-F'04) was born in Stockholm, Sweden, in 1961. He received the M.S. degree in electrical engineering and applied physics from Linköping University, Linköping, Sweden, in 1986 and the Ph.D. degree in electrical engineering from Stanford University, Stanford, CA, in 1989.

Dr. Ottersten has held research positions at the Department of Electrical Engineering, Linköping University, the Information Systems Laboratory, Stanford University, the Katholieke Universiteit Leuven, Leuven, and the University of Luxembourg. During 96/97, he was Director of Research at ArrayComm Inc, a start-up in San Jose, California based on Ottersten' s patented technology. He has co-authored journal papers that received the IEEE Signal Processing Society Best Paper Award in 1993, 2001, 2006, and 2013 and 3 IEEE conference papers receiving Best Paper Awards. In 1991, he was appointed Professor of Signal Processing at the Royal Institute of Technology (KTH), Stockholm. From 1992 to 2004, he was head of the department for Signals, Sensors, and Systems at KTH and from 2004 to 2008, he was dean of the School of Electrical Engineering at KTH. Currently, he is Director for the Interdisciplinary Centre for Security, Reliability and Trust at the University of Luxembourg. As Digital Champion of Luxembourg, he acts as an adviser to European Commissioner Neelie Kroes.

Dr. Ottersten has served as Associate Editor for the IEEE TRANSACTIONS ON SIGNAL PROCESSING and on the editorial board of IEEE Signal Processing Magazine. He is currently editor in chief of EURASIP Signal Processing Journal and a member of the editorial boards of EURASIP Journal of Applied Signal Processing and Foundations and Trends in Signal Processing. He is a Fellow of the IEEE and EURASIP and a member of the IEEE Signal Processing Society Board of Governors. In 2011, he received the IEEE Signal Processing Society Technical Achievement Award. He is a first recipient of the European Research Council advanced research grant. His research interests include security and trust, reliable wireless communications, and statistical signal processing. 